Royal Ontario Museum

Life Sciences

Miscellaneous Publication

\title{
Key to the Quaternary Pollen and Spores of the Great Lakes Region
}

John H. McAndrews, Albert A. Berti and Geoffrey Norris

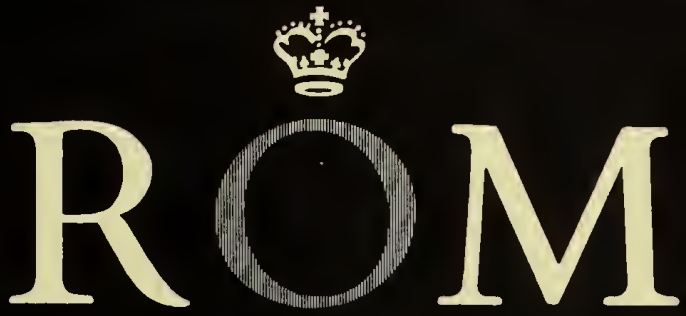



ROYAL ONTARIO MUSEUM

LIFE SCIENCES

MISCELLANEOUS PUBLICATION

JOHn H. McANDREws, Key to the Quaternary

albert a. Berti, Pollen and Spores

GEOFFREY NORRIS

of the

Great Lakes Region

Publication date: 9 November 1973

ISBN 0-88854-149-X

Suggested citation: Life Sci. Misc. Publ., R. Ont. Mus. 


\section{ROYAL ONTARIO MUSEUM \\ PUBLICATIONS IN LIFE SCIENCES}

The Royal Ontario Museum publishes three series in the Life Sciences:

LIFE SCIENCES CONTRIBUTIONS, a numbered series of original scientific publications, including monographic works.

LIFE SCIENCES OCCASIONAL PAPERS, a numbered series of original scientific publications, primarily short and usually of taxonomic significance.

LIFE SCIENCES MISCELLANEOUS PUBLICATIONS, an unnumbered series of publications of varied subject matter and format.

All manuscripts considered for publication are subject to the scrutiny and editorial policies of the Life Sciences Editorial Board, and to review by persons outside the Museum staff who are authorities in the particular field involved.

\section{LIFE SCIENCES EDITORIAL BOARD}

Chairman, R. L. PETERSEN

Editor, D. BARR

Associate Editor, J. C. BARLow

Associate Editor, J. R. TAMSITT

JoHN H. MCANDREws is Associate Curator of the Department of Geology, Royal Ontario Museum and Associate Professor in the Department of Botany, University of Toronto.

ALbert A. Berti is a National Research Council Fellow at the Department of Botany, University of Toronto and Research Associate of the Department of Geology, Royal Ontario Museum.

Geoffrey NORris is Associate Professor in the Department of Geology, University of Toronto and Research Associate of the Department of Geology, Royal Ontario Museum.

PRICE : $\$ 2.50$

(C)The Royal Ontario Museum, 1973

100 Queen's Park, Toronto, Canada

PRINTED AT THE UNIVERSITY OF TORONTO PRESS

LIBRARY

ROYAL ONTARIO MUSEUM 


\section{Contents}

Introduction, 1

Materials and methods, 1

Using the key, 3

Key to pollen classes, 4

Key to spore classes, 4

Key to pollen grains, 5

Tetrads, 5

Vesiculate, 5

Polyplicate, 5

Inaperturate, 5

Monoporate, 6

Diporate, 6

Triporate, 6

Stephanoporate, 7

Periporate, 7

Monocolpate, 8

Dicolpate, 8

Tricolpate, 8

Stephanocolpate, 10

Pericolpate, 10

Tricolporate, 10

Stephanocolporate, 12

Key to spores, 13

Alete, 13

Monolete, 13

Trilete, 13

Glossary of morphological terms, 15

Figures, 19

Acknowledgments, 60

Literature cited, 60 


\section{Digitized by the Internet Archive in 2012 with funding from Royal Ontario Museum}




\section{Key to the Quaternary Pollen and Spores of the Great Lakes Region}

\section{Introduction}

In recent years the method of fossil pollen and spore analysis (Faegri and Iversen, 1964) has become important in both paleoecological reconstructions of vegetation and climate and in biostratigraphic correlation of sediments. Effective reconstructions and correlations depend upon accurate fossil identifications.

Scattered information is available on pollen and spore morphology, but relatively little has been organized in a form that can be used for fossil identification in the Great Lakes region. These aids include Sears (1930), "Common fossil pollen of the Erie Basin," which contains a key to only 33 taxa, illustrated by drawings. Presently, pollen analysts commonly use the unillustrated key to the northwest European pollen types (Faegri and Iversen, 1964), but it is not wholly applicable to our region nor does it include spores. Likewise, the key of Kapp (1969), "How to know pollen and spores," covers only the United States, is illustrated by drawings and does not focus on fossil identification. Another geographically limited but useful aid is Richard's (1970) "Atlas pollinique des arbres et de quelques arbustes indigènes du Québec," which contains excellent photographs and descriptions but omits herb pollen and lacks a general key. The recent atlas of tree pollen by Adams and Morton (1972) has similar limitations, but its scanning electron micrographs are excellent for sculptural detail.

Fossil pollen assemblages are composed primarily of pollen of windpollinated species, especially trees, and to a lesser extent shrubs and herbs. Except for aquatic plants, pollen of insect-pollinated species are rare. Our photo-illustrated key is designed for the identification of fossil pollen and spores (microspores and homospores of pteridophytes and Sphagnum) commonly found in Quaternary deposits of the states and the province surrounding the Great Lakes. The key is most applicable in the middle Great Lakes region, especially southern Ontario and Michigan, but is also useful in such peripheral areas as the Atlantic Coast, and the subarctic and prairie regions as shown in Fig. 1.

\section{Materials and Methods}

The 144 entries in the key were selected from our analyses of sediments of late Pleistocene (Sangamonian, Wisconsinan) and Holocene age and on fossils reported by others. The majority of taxa (76) are rare, that is, they usually represent less than $1 \%$ of a total fossil spectrum. Aquatic taxa are also rare, but some may be locally abundant. Only 46 taxa are common enough to occur consistently with values greater than $1 \%$. Not all grow in the Great Lakes region today, but most grew in this region sometime in the 


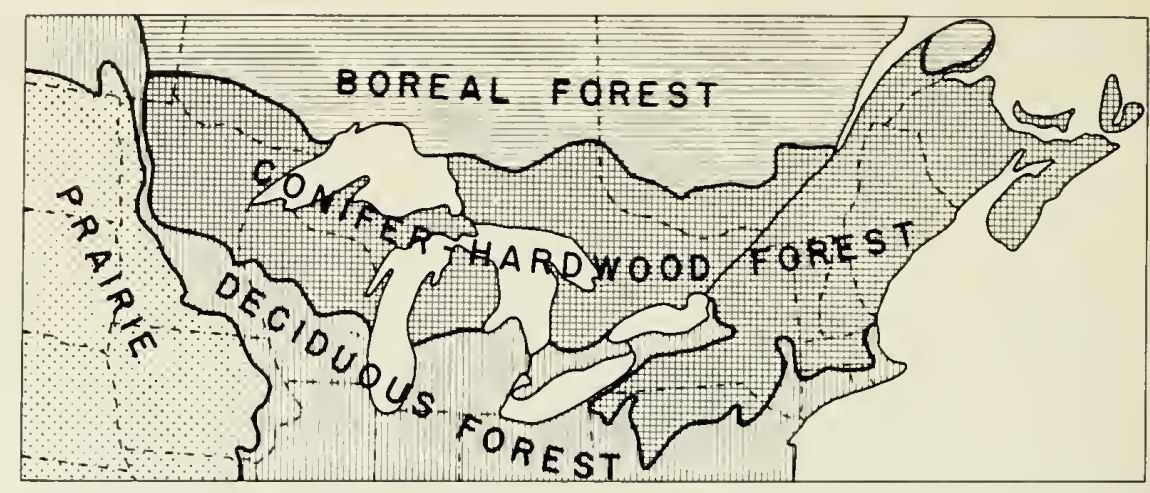

Fig. 1-Zonal vegetation map of the Great Lakes region (modified from Braun, 1950 , and Rowe, 1959). The key is most applicable in the conifer-hardwood forest zone.

Quaternary. Fossil Ephedra and Sarcobatus are exceptions, in that they appear to have been blown in from beyond our region (Maher, 1964).

The key is in two parts, one for the sixteen classes of pollen and the second for the three classes of spores. In our fossil flora, spores (Figs. 16-21) tend to be morphologically simpler than pollen (Figs. 2-15). Spores have a slitshaped aperture (laesura) that lacks a membrane. They may have a single laesura (monolete, Fig. 16c) or three triradiate laesurae (trilete, Fig. 18c). The only exception treated here is the alete Equisetum (Fig. 16A).

In pollen grains apertures may be absent (Fig. 5E), simple (elongate colpi, Fig. 10K, or circular pores, Fig. 5M), or compound (colpi with pores, Fig. $13 \mathrm{~N}$ ). Usually multiple apertures are present, and in fossils the apertures often retain a membrane (Figs. 9c,s; 10L). Spores commonly have a relatively thick, structureless wall, and many taxa possess a loose-fitting sac or perine that is often lost from fossil spores. Pollen have walls of varying thickness and complexity and may have easily distinguished columellae and a tectum (Fig. 13E); pollen never have a perine. The size of spores and pollen is generally similar except that the minimum size of pteridophyte spores is about $25 \mu \mathrm{m}$, whereas many pollen are smaller. Fungal spores (not considered in the key) can be confused with pollen and spores of vascular plants but are distinguishable by their small size $(5-20 \mu \mathrm{m})$, general lack of apertures, and simple wall structure.

We attempted to keep terminology to a minimum and have defined terms in the glossary (p. 15). Spore terminology follows Harris (1955), and pollen terminology is based on Faegri and Iversen (1964). More exhaustive and detailed treatments of pollen and spore morphology are found in texts such as Erdtman (1957, 1965, 1966), Erdtman and Sorsa (1971), Kremp (1965), and Tschudy and Scott (1969).

The key is illustrated with reference pollen and spores collected from herbarium sheets or living plants, treated according to the method of Faegri and Iversen (1964) with KOH and acetolysis solution, and mounted in silicone oil. Due to the inflated and uncompressed nature of the pollen and the 
shallow depth of focus of high magnification microscope objectives, it was not possible to illustrate all morphologic features in one photograph. In general, spores and pollen are best illustrated either in "optical section" to show features of the internal wall structure (Fig. 6F) or in surface view to show details of the external sculpture pattern (Fig. 6A). Occasionally, both the optical section and the surface pattern of the exine are illustrated in the same photograph (Fig. 6E). Only the most useful diagnostic features are illustrated in this work allowing the user to "key-out" an unknown grain with the aid of a light microscope.

Both polar and equatorial views have been used to illustrate the various morphologic features in this key; these views are indicated in the Figure captions by the abbreviations "pv" and "ev" respectively. Photograph magnifications are at X1000 except for the vesiculate genera Pinus, Picea, and Abies (Fig. 2) which are at X500.

Pollen and spore measurements in the Figure captions were made with an ocular micrometer on 10 undistorted grains from a single slide. Measurements are of the greatest dimension excluding sculptural projections and the perine. Measurements of vesiculate grains are of the body in equatorial view. The average size is given in $\mu \mathrm{m}$ with the range in parentheses.

In addition to reference slides, the most effective use of the key requires a high quality microscope, and good fossil preparations. Fossil pollen are often folded, broken or shrunk, and size measurements of reference pollen must be used with caution. Apertures are often obscured, and we recommend the use of a liquid mounting medium (silicone oil or glycerine) so that grains can be rolled to reveal key features.

In some cases the pollen or spore class to which a grain belongs (e.g. Equisetum, Ambrosia, Tilia or Cyperaceae) is either uncertain or difficult to determine. This problem has been overcome by entering the pollen or spore in more than one class. In addition, because the key is basically for identification rather than taxonomy, Equisetum and Selaginella selaginoides are entered in both the pollen and the spore classes.

\section{Using the Key}

Use of the key involves several steps. First the unknown is classified as a pollen grain or spore. Then it must be keyed to class with either the "Key to pollen classes" or the "Key to spore classes." The third step is to identify the unknown within the proper class in the "Key to pollen grains" or "Key to spores." Finally, the unknown specimen should be compared with the photographs and with reference material, to confirm or reject the identification.

Nomenclature follows the 8th edition of Gray's Manual of Botany (Fernald, 1950). Species names and authors that do not appear in that manual are Sarcobatus vermiculatus (Hook.) Torr., Iva axillaris Pursh, Ephedra aspera Engelm., E. coryi Reed, E. nevadensis Wats., E. torreyana Wats., E. trifurca Torr., and $E$. viridis Coville. 


\section{Key to Pollen Classes}

A. Grains in groups of 4

1 Tetrads p. 5

AA. Grain single (monads)

B. Apertures absent (or indistinct)

C. With two bladders

CC. Without bladders

D. With ridges separated by folds

DD. Without ridges and folds

BB. Apertures present

2 Vesiculate p. 5

3 Polyplicate p. 5

4 Inaperturate p. 5

E. Apertures simple, i.e. either pores or colpi

F. With pores
1 pore
2 pores
3 pores

More than 3 pores cquatorially arranged

5 Monoporate p. 6

6 Diporate p. 6

7 Triporate p. 6

8 Stephanoporate p. 7

More than 3 pores, some or all not equatorial

9 Periporate p. 7

FF. With colpi
1 colpus
2 colpi
10 Monocolpate p. 8
3 meridional colpi
11 Dicolpate p. 8
More than 3 meridional colpi
12 Tricolpate p. 8
More than 3 colpi, some or all not meridional

14 Pericolpate p. 10

EE. Apertures compound, i.e. pores in colpi

3 colpi meridionally arranged

15 Tricolporate p. 10

More than 3 colpi meridionally arranged

16 Stephanocolporate $\quad$ p. 12

\section{Key to Spore Classes}

A. Spore without apcrture

1 Alete p. 13

AA. Spore with slit-shaped aperture (laesura)

B. Spore with single laesura ...

2 Monolete p. 13

BB. Spore with triradiate laesurae

3 Trilete p. 13 


\section{Key to Pollen Grains}

\section{Tetrads}

A. Reticulate, tetrads usually rhombohedral, each grain monoporate. Typhaceae

Typha latifolia 2A, B

AA. Not reticulate, tetrahedral tetrads

B. Echinate

C. Spines 5-8 $\mu \mathrm{m}$ long and spaced 5-10 $\mu \mathrm{m}$ apart. Rare to locally abundant. Selaginellaceae ... Selaginella selaginoides 2C;19E

CC. Spines 2-3 $\mu \mathrm{m}$ long and more closely spaced. Rare. Droseraceae

Drosera 2D

BB. Psilate or scabrate, each grain tricolpate or tricolporate

Ericaceae 2E-J

2. Vesiculate Bladders with internal reticulum. Pinaceae

A. Bladders hemispherical (not constricted at attachment with body of grain), cap of body (proximal surfacc) of even thickness, body 50-80 $\mu \mathrm{m}$ broad. Reticulum of bladder finer than in Pinus (Fig. 3D) or Abies (Fig. 3F)

Picea 3A-D

AA. Bladders more than hemispherical (constricted at attachment to body), cap of uneven thickness

B. Body of grain $60-90 \mu \mathrm{m}$ broad, bladder $45-70 \mu \mathrm{m}$ broad, cap thick $(5 \mu \mathrm{m})$ but often appearing thinnest in centre Abies 3E, F

B.B. Body $40-55 \mu \mathrm{m}$ broad, bladder $24-40 \mu \mathrm{m}$ broad, cap thinner ( 2 $\mu \mathrm{m})$ and of even thickness except along margin. Pinus. Species separation by size statistics is not reliable (Whitehead, 1964).

C. Distal verrucae present and about $1 \mu \mathrm{m}$ broad. (Smaller and more scattercd verrucae occur on Picea). Section Haploxylon

Pinus strobus $3 \mathrm{~K}$

CC. Distal verrucae absent. Section Diploxylon ( $P$. banksiana, $P$. resinosa and $P$. rigida)

Pinus banksiana type 3G-J

3. Polyplicate (with meridional folds), prolate, grain c. $25 \times 45-65 \mu \mathrm{m}$. Ephedraceae

A. Grain with $c .15$ straight folds. Includes among others E. aspera and E. trifurca. Rare

Ephedra torreyana type $4 \mathrm{~A}$

AA. Grain with $c .6$ branched folds. Includes among others $E$. coryi and E. viridis. Rare

Ephedra nevadensis type $4 \mathrm{~B}$

\section{Inaperturate}

A. Grain banana-shaped, with an imperfect reticulum, grain $c .35 \times 70$ $\mu \mathrm{m}$. Rare. Zosteraceae

Ruppia 4c

AA. Grain spheroidal (or pear-shaped in Cyperaceae) but often distorted by splits and folds

B. Grain 55-85 $\mu \mathrm{m}$. Pinaceae

C. Psilate

Larix 4D

CC. With irregular, hollow vervucae

Tsuga 4E

BB. Grain 20-45 $\mu \mathrm{m}$ except some Cyperaceae as large as $60 \mu \mathrm{m}$ 
D. Gemmate with gemmae distinct but scattered, fossil grains often angularly broken. Cupressineae

Thuja or Juniperus $5 \mathrm{~A}-\mathrm{C}$

DD. Psilate, scabrate or reticulate

E. Psilate or scabrate-verrucate

F. Psilate, often with a wrinkled perine. Rare to locally abundant. Equisetaceae

Equisetum 5D; 16A

FF. Scabrate-verrucate

G. Spheroidal, no poroids and often folded or flattened. Rare to locally abundant. Salicaceae ....... Populus 5E

GG. Pear-shaped with 3 lateral poroids and 1 poroid on broad end. Most fossil grains are probably aquatic species of Carex, Scirpus, Eleocharis or Cyperus. See Faegri and Iverson (1964) for more detailed indentification

Cyperaceae 5F, G; 7L, M

EE. Reticulate

H. Reticulum broken (muri incomplete). Includes the other members of the subgenus Coleogeton, i.e. $P$. filiformis and $P$. vaginatus. Zosteraceae

Potamogeton pectinatus type $5 \mathrm{H}$

HH. Reticulum unbroken. Zosteraceae or Juncaginaceae ....

Potamogeton natans

type (subgenus Eupotamogeton) or Triglochin 5I, J

\section{Monoporate}

A. Pore with distinct annulus, psilate or scabrate, grain c. 20-50 $\mu \mathrm{m}$ (larger in cereals, e.g. Zea Fig. 5M)

Gramineae $5 \mathrm{~K}-\mathrm{M}$

AA. Pore without annulus, grain $18-30 \mu \mathrm{m}$

B. Reticulate. Sparganiaceae or Typhaceae

BB. Echinate. Rare. Lemnaceae

Sparganium or Typha angustifolia 6A, B

6. Diporate

A. Psilate, pore circular and annulate, exine thin, grain spheroidal, 18-20 $\mu \mathrm{m}$. Rare. Moraceae

Morus 6D; 7D

AA. Psilate, but with protruding pores, exine thicker

rare grains of Corylaceae

7. Triporate Grains of Ostrya and Carpinus occasionally have 4 pores.

A. Pores distinctly protruding, psilate

B. Grain $45-80 \mu \mathrm{m}$. Rare. Onagraceae

Epilobium 6E

BB. Grain $16-35 \mu \mathrm{m}$

C. Inner surface of pore margin rough. Myricaceae

Comptonia or Myrica $6 \mathrm{~F}$

CC. Inner surface of pore margin smooth. Corylaceae

D. With separation of endexine at base of pore, exine thick

Betula 6G-I

DD. Without separation of endexine, exine thinner

Ostrya or Carpinus $6 \mathrm{~J}-\mathrm{K}$

AA. Pores scarcely protruding 
E. Reticulate, endexine conspicuously thickened around pore, oblate. Tiliaceae

Tilia $6 \mathrm{~L} ; 14 \mathrm{M}$

EE. Psilate-scabrate

F. Annulate, spheroidal

G. Pore sunken, i.e. tectum swells outward at pore and bends inward at inner margin, psilate. Rare. Cannabinaceae

Humulus $6 \mathrm{M}$

GG. Pore not sunken, scabrate. Rare. Ulmaceae .... Celtis 6N, o

FF. Not annulate, suboblate-oblate

H. Grain $40-55 \mu \mathrm{m}$, slightly heteropolar. Juglandaceae

HH. Grain $<40 \mu \mathrm{m}$

Carya 7A

I. Grain $20-30 \mu \mathrm{m}$, pores equatorial and slightly protruding, exine thick. Corylaceae

Corylus 78

II. Grain $12-20 \mu \mathrm{m}$, psilate, pores not protruding, exine thin $(0.5 \mu \mathrm{m})$. (Exceptional triporate Morus, Fig. 7D, will key out here.) Rare. Urticaceae

Urtica 7c

\section{Stephanoporate}

A. Arci (internal thickenings) connecting the 4 or 5 (rarely 3 or 6 ) protruding pores. Corylaceae

Alnus $7 \mathrm{E}-\mathrm{G}$

AA. Without arci

B. Rugulate-reticulate, 4-6 non-protruding and non-annulate pores, oblate. Ulmaceae

Ulmus $7 \mathrm{H}, \mathrm{I}$

BB. Psilate-scabrate, 4 (3-6) non-protruding but strongly annulate pores. (Ostrya and Carpinus occasionally have 4 protruding pores). Haloragaceae

C. Annulus $2-3 \mu \mathrm{m}$ high, pores asymmetrically situated. Rare Myriophyllum alterniflorum $7 \mathrm{~J}$

CC. Annulus 1-2 $\mu \mathrm{m}$ high, pores symmetrically situated. Includes among others $M$. farwellii, $M$. spicatum, $M$. verticillatum Myriophyllum exalbescens type $7 \mathrm{~K}$

\section{Periporate}

A. Heteropolar (pores mostly concentrated on one hemisphere)

B. Pear-shaped, scabrate, with 3 (4-6) lateral poroids (thin areas) and 1 poroid on broad end

Cyperaceae $5 \mathrm{~F}, \mathrm{G} ; 7_{\mathrm{L}}, \mathrm{M}$

BB. Oblate, psilate, 7-18 annulate pores. Juglandaceae

C. $7-11$ pores

CC. $12-18$ pores

AA. Not heteropolar, spheroidal

D. Echinate with spines $2 \mu \mathrm{m}$ long and regularly spaced, pores indistinct. Alismataceae

Sagittaria 7Q

DD. Not echinate

E. Reticulate, grain $30-50 \mu \mathrm{m}$

F. Reticulum coarse (lumina $5-10 \mu \mathrm{m}$ ), pores at bottom of lumina. Includes among others $P$. hydropiper, $P$. hydropiperoides, $P$. pensylvanicum, $P$. punctatum and $P$. sagittatum. Rare. Polygonaceae ..... Polygonum lapathifolium type 8A 
FF. Reticulum finer (lumina $1 \mu \mathrm{m}$ ). Hamamelidaceae

Liquidambar 8B

EE. Psilate-scabrate or verrucate

G. Distinctly annulate, grain 15-30 $\mu \mathrm{m}$

H. More than 16 pores, exine thick. Rare

HH. Fewer than 16 pores

Caryophyllaceae $8 \mathrm{C}$

I. About 14 pores, psilate. Rare. Chenopodiaceae

Sarcobatus 8D

II. 8-14 pores, scabrate. Mostly restricted to sediments of the past century. Plantaginaceae

Plantago lanceolata $8 \mathrm{E}$

GG. Not distinctly annulate

J. More than 30 pores. Includes Amaranthaceae and Chenopodiaceae except Sarcobatus. For method of distinguishing species by pore number see McAndrews and Swanson (1967)

Chenopodiineae p.p. $8 \mathrm{~F}, \mathrm{G}$

JJ. Fewer than 30 pores

$\mathrm{K}$. Pore margin distinct, exine very thick, grain $>30 \mu \mathrm{m}$.

Rare

Caryophyllaceae

KK. Pore margin indistinct, grain 15-30 $\mu \mathrm{m}$

L. Psilate, 6-12 pores. Rare. Ranunculaceae

Thalictrum $8 \mathrm{H}$

LL. Verrucate, 4-11 pores. Mostly restricted to sediments of this century. Includes among others $P$. virginica and $P$. pusilla. See also Bassett and Crompton (1968). Rare

Plantago major type 8I

10. Monocolpate Nymphaeaceae

A. Colpus circular, baculate

Nymphaea $8 \mathrm{~J}, \mathrm{~K}$

AA. Colpus linear

B. Echinate, spines 5-6 $\mu \mathrm{m}$ long

BB. Psilate-scabrate. Rare

Nuphar $8 \mathrm{~L}$

Brasenia $8 \mathrm{M}$

11. Dicolpate, scabrate-verrucate, 50-72 $\mu \mathrm{m}$. Rare.

Pontederiaceae

Heteranthera $8 \mathrm{~N}$

\section{Tricolpate}

A. Sculpturing elements elongated parallel to surface

B. Striate or rugulate
C. Striate

D. Colpus without membrane, $>30 \mu \mathrm{m}$. For detailed treatment of Acer pollen see Helmich (1963).

Aceraceae

Acer rubrum 9A, B

DD. Colpus with verrucate membrane, $<30 \mu \mathrm{m}$. Rare. Saxifragaceae ..... Saxifraga oppositifolia 9C, D

CC. Rugulate

E. Grain $>30 \mu \mathrm{m}$, delicately rugulate-reticulate, with colpus membrane. Aceraceae

Acer saccharinum 9E, F

EE. Grain $<30 \mu \mathrm{m}$ 
F. With colpus membrane, endexine thin. Rare. Rosaceae

Dryas 9G-I

FF. Colpus membrane absent, endexine thick. Rare. Acer-

BB. Reticulate aceae

Acer negundo $9 \mathrm{~J}-\mathrm{L}$

G. Colpus with psilate-scabrate margin, polar area small

H. Subprolate-prolate, without operculum. Salicaceae

I. Reticulum unbroken (muri complete) Salix p.p. 9M-P

II. Reticulum broken. Rare Salix herbacea 9Q, R

HH. Prolate-perprolate, grain c. $25 \times 45 \mu \mathrm{m}$, with operculum, reticulum fine, Rare. Leguminosae

Petalostemum purpureum 9s

GG. Margin absent, i.e. reticulum of exine extends to edge of colpus

J. Tectate, polar area small

$\mathrm{K}$. Exine thick $(2.2 \mu \mathrm{m})$, lumina $1.5 \mu \mathrm{m}$, colpus membrane absent. Aceraceae Acer saccharum 9T, U

KK. Exine thinner, lumina smaller. Includes among others

JJ. Intectate Stachys and Scutellaria. Rare. Labiatae p.p. 10A, B

L. Polar area small, exine thick $(2.5 \mu \mathrm{m})$, lumina $1.5 \mu \mathrm{m}$, Rare ...... ... Cruciferae 10c, D

LL. Polar area medium to large, exine thinner $(<1.5 \mu \mathrm{m})$, lumina smaller $(<1.5 \mu \mathrm{m})$

M. Colpus membrane absent, 20-25 $\mu \mathrm{m}$. Oleaceae

Fraxinus nigra or F. quadrangulata $10 \mathrm{E}-\mathrm{G}$

MM. Colpus with scabrate membrane, spherical, grain 16$20 \mu \mathrm{m}$. Platanaceae

Platanus $10 \mathrm{H}$

AA. Sculpturing elements absent or isodiametric, or elongated perpendicular to surface (not striate, rugulate or reticulate)

N. Scabrate, spheroidal-subprolate

O. Irregularly scabrate, no colpus membrane, large polar area, grain 20-35 $\mu \mathrm{m}$. Fagaceae

Quercus 10I-K

OO. Very regularly scabrate, colpus membrane scabrate, small polar area, grain 22-26 $\mu \mathrm{m}$. Rare. Ranunculaceae Caltha 10r

NN. Not scabrate

P. Echinate, spines short (c. $1 \mu \mathrm{m})$, polar area large, spherical, grain 18-23 $\mu \mathrm{m}$. Compositae Ambrosia 10M;12M, N

PP. Verrucate or clavate

Q. Verrucate, grain 26-32 $\mu \mathrm{m}$. Rare. Includes species of the section Batrachium. Ranunculaceae

QQ. Clavate

Ranunculus flabellaris type $10 \mathrm{~N}$

R. Colpus with margin of smaller clavae, prolate, grain 22-32 $\mu \mathrm{m}$. Rare. Aquifoliaceae Ilex or Nemopanthus $10 \mathrm{o}, \mathrm{P}$

RR. Colpus without special margin, clavae-verrucae of various sizes, subprolate, grain 30-36 $\mu \mathrm{m}$. Rare. Rosaceae .....

Rubus chamaemorus $10 \mathrm{Q}, \mathrm{R}$ 


\section{Stephanocolpate}

A. Psilate-scabrate

B. Small polar area, 6-10 colpi. Rare. Rubiaceae Galium 10s-U

BB. Large polar area, 4 (5-6) colpi each with a broad membrane.

Rare. Hippuridaceae

AA. Reticulate or echinate

C. Reticulate

D. 6 colpi, small polar area. Rare. Includes among others Lycopus, Mentha, Monarda, and Prunella ….. Labiatae p.p. 11в, С

DD. 4 colpi, large polar area

E. Subprolate, reticulum fine, grain 20-24 $\mu \mathrm{m}$. Oleaceae

Fraxinus pennsylvanica or $F$. americana $11 \mathrm{D}-\mathrm{F}$

EE. Grain with shape of rectangular prism, reticulum mediumcoarse, 4 short colpi, grain c. $32 \mu \mathrm{m}$. Rare. Balsaminaceae

Impatiens $11 \mathrm{G}, \mathbf{H}$

CC. Echinate, 3 long and 3 short colpi. Rare. Loranthaceae

Arceuthobium 11I, J

\section{Pericolpate}

A. Reticulate, with clavae at bottom of lumina. Rare. Polygonaceae Polygonum amphibium or $P$. coccineum $11 \mathrm{~K}, \mathrm{~L}$ AA. Echinate. Rare. Portulacaceae Portulaca oleracea $11 \mathrm{M}$

15. Tricolporate This is the largest class and somewhat difficult because pores are often obscure. Reference slides are especially important.

A. Echinate. Compositae

B. Exine structure consisting of lacunae separated by echinate ridges arranged in a geometrical pattern. Rare

subfamily Liguliflorae $12 \mathrm{~A}-\mathrm{C}$

BB. Exine without lacunae or ridges. Subfamily Tubuliflorae

C. Spines $>1.5 \mu \mathrm{m}$ polar area small. Includes all genera except the Ambrosia group and Artemisia . Tubuliflorae p.p. 12D-G

CC. Spines short $(<1.5 \mu \mathrm{m})$, pores indistinct, grains 14-26 $\mu \mathrm{m}$. Ambrosia group

D. Columellae of tectum distinct, colpi indistinct, polar area large E. Exine thick, spines $c .1 \mu \mathrm{m}$ long. Rare to locally abundant

EE. Exine thinner, spines shorter. Rare Iva ciliata $12 \mathrm{H}, \mathbf{I}$
Xanthium $12 \mathrm{~J}$

DD. Columellae indistinct

F. Colpi long and distinct, small polar area. Rare to locally abundant

Iva xanthifolia $12 \mathrm{~K}, \mathrm{~L}$

FF. Colpi short and indistinct, large polar area. The predominantly western Iva axilaris is identical

Ambrosia 10M; 12M, N

AA. Not echinate

G. Psilate-scabrate

H. Grain $<16 \mu \mathrm{m}$, prolate, pores equatorially elongated. Fagaceae Castanea 120, P 
HH. Grain $>18 \mu \mathrm{m}$

I. Oblate, triangular in polar view, pores protruding. Elaeagnaceae

J. Colpi short $(c .8 \mu \mathrm{m})$. Rare

Elaeagnus $13 \mathrm{~A}$

JJ. Colpi long $(c .20 \mu \mathrm{m})$. Rare. Shepherdia argentea 13в

II. Spheroidal to prolate or perprolate

K. Spheroidal to prolate

L. Equatorial thickening of endexine. Includes among others $P$. cristatum and P. achoreum. Rare. Polygonaceae

LL. No equatorial thickening

Polygonum aviculare type $13 \mathrm{C}, \mathrm{D}$

M. Grains spheroidal

N. Exine thick (3-4 $\mu \mathrm{m})$ but thinning toward colpus, columellae distinct, micro-echinate, grain 20-30 $\mu \mathrm{m}$. Compositae . . Artemisia 13E, F

NN. Exine thinner and of uniform thickness

O. Pore protruding slightly, circular and annulate, grain 30-34 $\mu \mathrm{m}$. Rare. Nyssaceae

OO. Pore not protruding

Nyssa 13G-I

P. Grain $35-43 \mu \mathrm{m}$, scabrate, pore of ten elongated along meridian and often not very distinct. Fagaceae .............. Fagus 13J, K

PP. Grain 25-30 $\mu \mathrm{m}$, pore circular and distinct. Rare to locally abundant. Polygonaceae

Rumex 13L, M

MM. Grains prolate

Q. Pore circular and slightly annulate, grain $24 \times 35$ $\mu \mathrm{m}$. Rare to locally abundant. Elaeagnaceae

QQ. Pore not circular

Shepherdia canadensis $13 \mathrm{~N}, \mathrm{o}$

R. Pore not elongated but characterized by a constriction of the colpus. Cornaceae

S. Grain $<25 \mu \mathrm{m}$, psilate. Rare

Cornus canadensis $13 \mathrm{P}, \mathrm{Q}$

SS. Grain $>35 \mu \mathrm{m}$. Rare. Includes among others

$C$. racemosa, $C$. alternifolia and $C$. rugosa

Cornus stolonifera type $14 \mathrm{~A}-\mathrm{C}$

RR. Pore elongated equatorially, exine thicker at poles than at equator, grain $c .35 \times 50 \mu \mathrm{m}$. Rare. Polygonaceae

Polygonum viviparum $14 \mathrm{D}, \mathrm{E}$

KK. Perprolate, exine of varying thickness, pore elongated equatorially. Rare.

Umbelliferae $14 \mathrm{~F}-\mathrm{H}$

GG. Striate or reticulate

T. Striate 
U. Striae very pronounced and parallel with meridional orientation, transverse colpus absent. Rare. Gentianaceae

Menyanthes 14I, J

UU. Striae finer, pore indistinct, transverse colpus present or absent. Includes among others Potentilla and Prunus

Rosaceae p.p. $14 \mathrm{~K}, \mathrm{~L}$

TT. Reticulate

V. Oblate, endexine conspicuously thickened around pore, colpi short and indistinct, ca. $36 \mu \mathrm{m}$. Tiliaceae . Tilia $6 \mathrm{~L} ; 14 \mathrm{M}$

VV. Spherical to prolate, endexine not conspicuously thickened around pore, colpi longer and distinct

W. Pore large (about $1 / 2$ diameter of spheroidal grain), colpus with an operculum, grain 20-22 $\mu \mathrm{m}$. Rare. Leguminosae

WW. Pore smaller

Amorpha $14 \mathrm{~N}, \mathrm{o}$

$\mathrm{X}$. Colpus margin psilate, reticulum fine, prolate, pore not pronounced. Salicaceae $\quad$ Salix p.p. 14P, Q $\mathrm{XX}$. No distinct colpus margin

Y. Pore an equatorial constriction of the colpus, grain 15-20 $\mu \mathrm{m}$. Rare. Caprifoliaceae ..... Sambucus 15A YY. Pore circular and annulate

Z. Pore wider than colpus, micro-reticulate. Rare. Vitaceae

Vitis $15 \mathrm{~B}, \mathrm{C}$

ZZ. Pore not as wide as colpus, reticulum coarser. Rare to locally abundant. Rubiaceae

Cephalanthus $15 \mathrm{D}, \mathrm{E}$

\section{Stephanocolporate}

A. With c. 15 colpi, pores fused together around the equator. Rare. Lentibulariaceae

Utricularia $15 \mathrm{~F}, \mathrm{G}$

AA. With 6 colpi that form 3 pairs, each pair of colpi within an equatorially elongated pore. Rare. Rosaceae

Sanguisorba canadensis $15 \mathrm{H}, \mathrm{I}$ 


\section{Key to Spores}

1. Alete Perine wrinkled and grain often broken. Rare to locally abundant. Equisetaceae

Equisetum 5D;16A

2. Monolete

A. Perine absent. Polypodiaceae

B. Psilate. Includes among others Asplenium trichomanes, Cystopteris fragilis, Dryopteris spinulosa and Onoclea sensibilis

BB. Verrucate

Polypodiaceae p.p. 16B, C

C. Grain $<42 \mu \mathrm{m}$, verrucae small and scattered. Rare

Athyrium filix-femina 16D

CC. Grain $>44 \mu \mathrm{m}$, verrucae larger and dense. Rare

Polypodium virginianum $16 \mathrm{E}$

AA. Perine present

D. Perine psilate-scabrate

E. Perine ragged in the region of the laesura. Rare to locally abundant. Isoetaceae

Isoetes $16 \mathrm{~F}$

EE. Not ragged in the region of the laesura. Rare. Polypodiaceae

Pteretis pensylvanica 16G

DD. Perine variously sculptured, not psilate-scabrate

F. Perine not echinate

G. Perine reticulate and loose fitting. Rare. Polypodiaceae

Woodsia ilvensis $16 \mathrm{H}$

GG. Perine variously ridged and folded. Includes among others Athryium thelypteroides, Dryopteris thelypteris and $D$. goldiana

Polypodiaceae p.p. 16I, J

FF. Perine echinate. Polypodiaceae

H. Echinae projecting from a tight-fitting perine

I. Echinae sharp and up to $6 \mu \mathrm{m}$ long. Rare

II. Echinae blunt and 2-4 $\mu \mathrm{m}$ long. Rare

Cystopteris fragilis $16 \mathrm{~L}$

Cystopteris bulbifera $16 \mathrm{~K}$

$\mathrm{HH}$. Perine loose-fitting, echinae on ridges

J. Echinae c. $1 \mu \mathrm{m}$ long. Rare ... Dryopteris spinulosa $17 \mathrm{~A}$

JJ. Echinae 2-4 $\mu \mathrm{m}$ long and very irregular. Rare

Camptosorus or Polystichum lonchitis 178, C

\section{Trilete}

A. With perine

B. Perine $70-85 \mu \mathrm{m}$ with folds and thickenings forming an imperfect reticulum. Rare. Polypodiaceae … Cheilanthes $17 \mathrm{D}, \mathrm{H}$

BB. Perine $30-55 \mu \mathrm{m}$

C. Perine large $(40-55 \mu \mathrm{m})$ loose-fitting and densely verrucate. Rare to locally abundant. Selaginellaceae 
CC. Perine smaller $(30-55 \mu \mathrm{m})$ tight-fitting and densely verrucate. Polypodiaceae

AA. Without perine

Pteridium aquilinum 17F, G

D. With negative sculptural elements or only psilate-scabrate

E. Psilate or scabrate. Polypodiaceae

F. Spore $>45 \mu \mathrm{m}$, psilate. Rare

FF. Spore $<45 \mu \mathrm{m}$, scabrate

G. Laesurae with lips, exine $>1.5 \mu \mathrm{m}$ thick. Rare

Adiantum or Dennstaedtia $18 \mathrm{~A}, \mathrm{~B}$

GG. Laesurae simple, exine $<1.5 \mu \mathrm{m}$ thick

Cheilanthes $17 \mathrm{D}, \mathrm{H}$

Pteridium aquilinum $18 \mathrm{C}, \mathrm{D}$

EE. Foveolate or fossulate

H. Foveolate. Lycopodiaceae

I. Spore $<25 \mu \mathrm{m}$, sides of spore concave in polar view. Rare Lycopodium lucidulum $18 \mathrm{E}, \mathrm{F}$

II. Spore $>30 \mu \mathrm{m}$ and nearly triangular in polar view. Rare

Lycopodium selago $18 \mathrm{G}, \mathbf{H}$

HH. Fossulate. Rare. Ophioglossaceae

Botrychium multifidum or B. dissectum 18I, J-L

DD. With positive sculptural elements, not scabrate

J. Sculptural elements radially elongated (baculate or echinate)

K. Baculae of varying size, spore spheroidal. Osmundaceae

L. Baculae about twice as long as broad and not fused. Rare

Osmunda cinnamomea 19A

LL. Paculae broader and some fused to form short ridges. Rare.. Osmunda regalis or O. claytoniana 19B-D

KK. Echinate with spines pointed or truncate and 5-8 $\mu \mathrm{m}$ long, spores often in tetrads. Rare to locally abundant. Selaginellaceae

Selaginella selaginoides $2 \mathrm{C}, 19 \mathrm{E}$

JJ. Sculptural elements elongated parallel to surface

M. Rugulate or reticulate

N. Rugulate, distal face with zig-zag ridges and proximal face verrucate. Rare. Lycopodiaceae

NN. Reticulate (at least on distal surface)

Lycopodium inundatum 19F, G

O. Muri with a broad base (tapering in cross-section). Rare. Ophioglossaceae ….......... Ophioglossum 20A, B

OO. Muri with vertical walls. Lycopodiaceae

P. Reticulum extending well onto proximal surface

Q. Nodes at angles of muri. Rare

QQ. Without nodes. Rare

Lycopodium clavatum 20C, D

Lycopodium

complanatum or L. tristachyum $20 \mathrm{E}-\mathrm{H}$

PP. Reticulum not extending well onto proximal surface

R. Laesurae about two-thirds spore radius, lumina small (up to $7 \mu \mathrm{m}$ ). Rare

Lycopodium obscurum 20I, J 
RR. Laesurae reaching equator, lumina larger (up to $10 \mu \mathrm{m})$. Rare

MM. Verrucate

Lycopodium annotinum $21 \mathrm{~A}, \mathrm{~B}$

S. Laesurae without lips, verrucae up to $6 \mu \mathrm{m}$ broad, spore 30-36 $\mu \mathrm{m}$. Rare. Ophioglossaceae

Botrychium virginianum 21C, D

SS. Laesurae with lips

T. Spore $<35 \mu \mathrm{m}$. Sphagnaceae . ..... Sphagnum 21E, F

TT. Spore $>35 \mu \mathrm{m}$. Rare. Includes among others Botrychium lunaria and $B$. lanceolatum. Ophioglossaceae

Botrychium simplex type $21 \mathrm{G}-\mathrm{K}$

\section{Glossary of Morphological Terms}

Terms are mostly defined in their singular noun form; adjectival forms are indicated with the suffix "ate".

AMB: outline of a pollen grain or spore in polar view (Betula Fig. 6G-I; Adiantum Fig. $18 \mathrm{~A}$ ).

ANNULUS: area surrounding a pore characterized by a thickening of the pollen wall (Caryophyllaceae Fig. 8C, D; Gramineae Fig. 5K-M).

APERTURE: a thinning or break in the pollen or spore wall that functions as a site for the emergence of the pollen tube or gametophyte.

ARCUS: elongate, narrow, curved thickening ( $\operatorname{arc}$ ) of the exine not parallel to the edges of the apertures (Alnus Fig. 7E, F).

BACULUM: sculptural element with radial projection isodiametric, at least one dimension $>1 \mu \mathrm{m}$, height $>$ width, sides parallel and top not pointed (Nymphaea Fig. 8J, $\mathrm{K}$ ).

CAP: the thickened proximal surface of the body of vesiculate grains (Picea Fig. 3A, C).

CLAVA: sculptural element with radial projection isodiametric, at least one dimension $>1 \mu \mathrm{m}$, height $>$ width, base constricted and top rounded (Nemopanthus Fig. 10P).

COLPUS: an aperture, arbitrarily defined as one with a length-breadth ratio $\geqslant 2.0$, the ends more or less acute (Quercus Fig. 10K).

COLUMELLA: radial rod of the exine separating a basal layer from the outer tectum (Artemisia Fig. 13E).

DISTAL: that part of a pollen or spore which faces outward in its tetrad, representing for example, in monoporate and monocolpate pollen, the area around the aperture.

ECHINUS: sculptural element with radial projection isodiametric, at least one dimension $>1 \mu \mathrm{m}$, height $>$ width, and pointed (Agoseris Fig. 12A-C: Helianthus Fig. 12D).

EKTEXINE: the outer part of the exine, consisting of a basal layer, radial columellae and an outer tectum. 
ENDEXINE: the innermost part of the exine, a relatively homogeneous zone below the ektexine.

EQUATOR: the imaginary line midway between the two poles that divides the grain into two polar hemispheres.

EXINE: the highly resistant wall surrounding the cytoplasm of pollen and spores, divisible into two main layers, the inner endexine and the outer ektexine.

FOSSULATE: sculptural type with surface of exine grooved (Botrychium multifidum (Fig. 18I, J).

FOVEOLATE: sculptural type with surface of exine pitted $>1 \mu \mathrm{m}$ diameter (Lycopodium lucidulum Fig. 18E).

GEMMA: sculptural element with radial projection isodiametric, at least one dimension $>1 \mu \mathrm{m}$, height $\leqslant$ width, base constricted and top rounded (Juniperus Fig. $5 \mathrm{~B})$.

HETEROPOLAR: apertures not distributed with equal density over the distal and proximal hemispheres (Juglans Fig. $7 \mathrm{~N}-\mathrm{P}$ ).

INTECTATE: pollen in which the ektexine elements, if present, are free and isolated (Ilex Fig. 10o), or form an open pattern (Fraxinus nigra Fig. 10E, F).

LACUNA: depressed area in the ektexine, few in number and regularly spaced in relation to the symmetry of the pollen grain (Agoseris Fig. 12A-C); c.f. lumen.

LAESURA: aperture of spores functioning as a dehiscence fissure.

LIP: a swelling or thickening along the margin of a laesura (Adiantum Fig. 18A); c.f. annulus.

LUMEN: the space bounded by the muri (walls) of a reticulum, not regularly spaced (Impatiens Fig. 11G, H); c.f. lacuna.

MEMBRANE: exine that forms the floor of an aperture (Saxifraga oppositifolia Fig. 9c, D).

MURUS: wall of the network that together with the lumina forms a reticulum (Impatiens Fig. $11 \mathrm{G}, \mathrm{H}$ ).

OBLATE: shape class with ratio between the polar and equatorial axes less than 0.75 (Ulmus Fig. 7H).

OPERCULUM: an isolated part of the ektexine that is contained within a colpus or pore (Amorpha canescens Fig. 14N, o).

PERINE: loose-fitting sac enclosing certain spore taxa (Pteretis pensylvanica Fig. 16G).

PERPROLATE: shape class with ratio between polar and equatorial axes $\geqslant 2.0$ (Umbelliferae Fig. 14F-H).

POLAR AREA INDEX: greatest distance between the ends of two colpi expressed as a ratio of the greatest breadth of the grain.

POLE: that part of a pollen grain or spore turned either inward or outward in its tetrad, characterized for example in the tricolpate class by the area between the ends of the colpi.

PORE: an aperture, arbitrarily defined as one with a length breadth ratio $<2.0$ (Juglans Fig. 7N-P).

POROID: pore-like, a poorly-defined pore (Cyperaceae Fig. 7L, M).

p.p.: pro parta, in part.

PROLATE: shape class with ratio between polar and equatorial axes $1.33-2.0$ (Shepherdia canadensis Fig. 13N, o).

PROXIMAL: that part of a pollen grain or spore which faces inward in its tetrad, representing for example in trilete spores the area around the triradiate laesurae. 
PSILATE: sculptural type with elements absent, exine smooth (Larix Fig. 4D). RETICULUM: net-like sculpturing with diameter of lumina $>$ breadth of muri (Lycopodium obscurum Fig. 20J).

RHOMBOHEDRAL: shape of a tetrad that approximates a rhombus (Typha latifolia Fig. 2A).

RUGULA: sculptural element tangentially elongated and with an irregular distribution (Lycopodium inundatum (Fig. 19F, G).

SCABRA: sculptural element with radial projection isodiametric and no dimension $\geqslant 1 \mu \mathrm{m}$ (Cyperaceae Fig. 7L, M).

STRIA: sculptural element tangentially elongated and more or less parallel to another stria (Acer rubrum Fig. 9A, B).

SUBOBLATE: shape class with ratio between polar and equatorial axes $0.88-$ 0.75 .

SUBPROLATE: shape class with ratio between polar and equatorial axes $1.33-$ 1.14 (Salix herbacea Fig. 90, R).

TECTATE: exine structure where tectum covers most of the surface of the grain.

TECTUM: outer layer (roof) of the exine that is most easily observed when separated from the endexine by a cavity (Artemisia Fig. 13E, F).

TETRAD: the four pollen grains or spores that are the product of a mother cell; although pollen or spores of most species separate and are dispersed as monads, some disperse as tetrads in a tetrahedral arrangement (Ericaceae Fig. 2E, J; Selaginella selaginoides Fig. 2c), or a rhombohedral arrangement (Typha latifolia Fig. 2A).

VERRUCA: sculptural element with radial projection more or less isodiametric, at least one dimension $>1 \mu \mathrm{m}$, height $\leqslant$ width, base not constricted, and top not pointed (Plantago major Fig. 81). 

FIGURES 
Fig. 2-Class 1, Tetrads, X1000 (p. 5)
A. Typha latifolia, rhombohedral tetrad
B. T. latifolia, irregular tetrad
C. Selaginella selaginoides
D. Drosera rotundifolia
E. Kalmia polifolia
F. K. polifolia
G. Chamaedaphne calyculata
H. Arctostaphylos uva-ursi
I. Andromeda glaucophylla
J. Vaccinium uliginosunt

$39.6(38-42) \mu \mathrm{m}$

49.8(46-54) $\mu \mathrm{m}$ $46.6(42-52) \mu \mathrm{m}$ $29.2(28-30) \mu \mathrm{m}$

$28.1(26-30) \mu \mathrm{m}$ $45.2(44-50) \mu \mathrm{m}$ 40.3(38-47) $\mu \mathrm{m}$ $38.2(34-40) \mu \mathrm{m}$ 
FIGURE 2
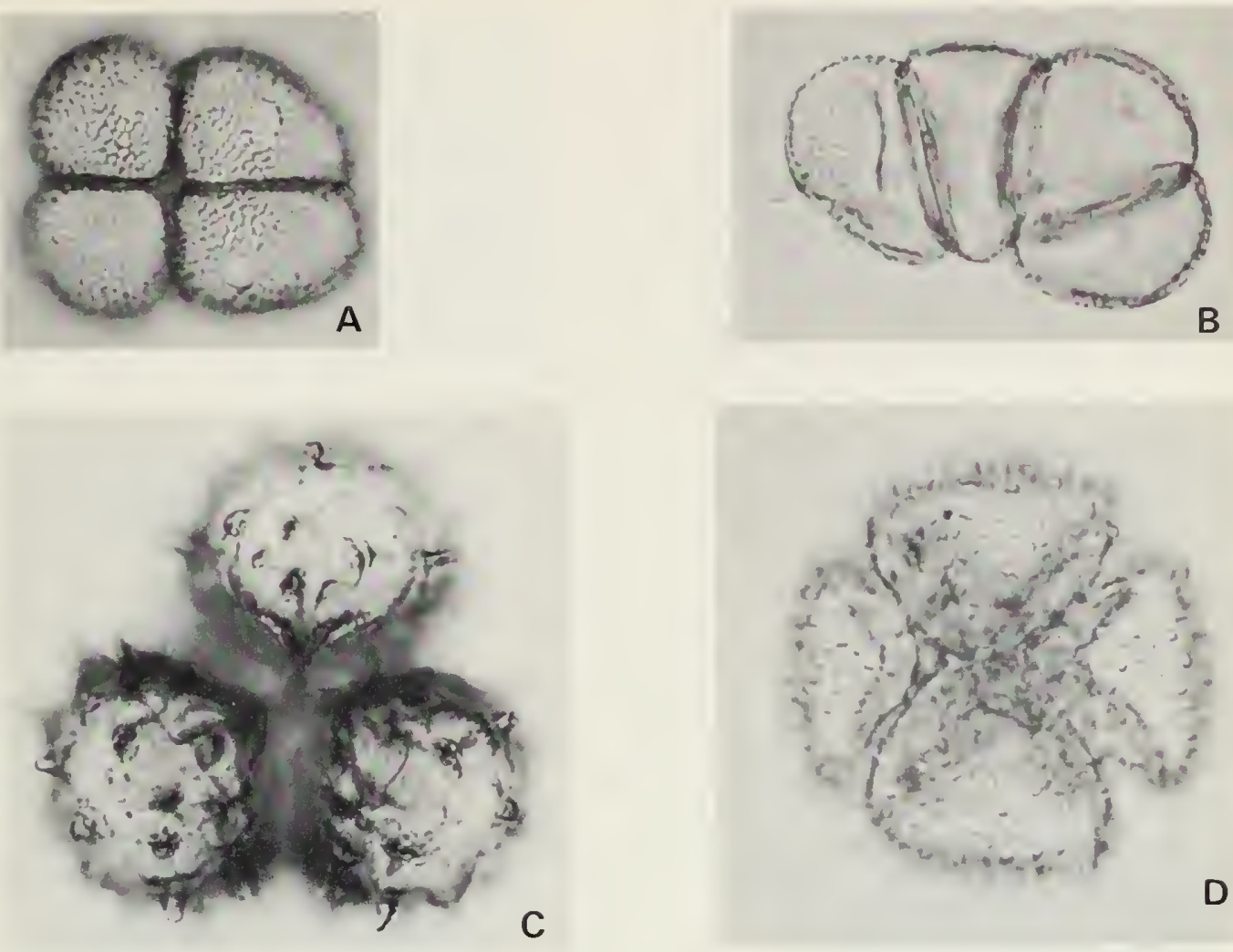

C
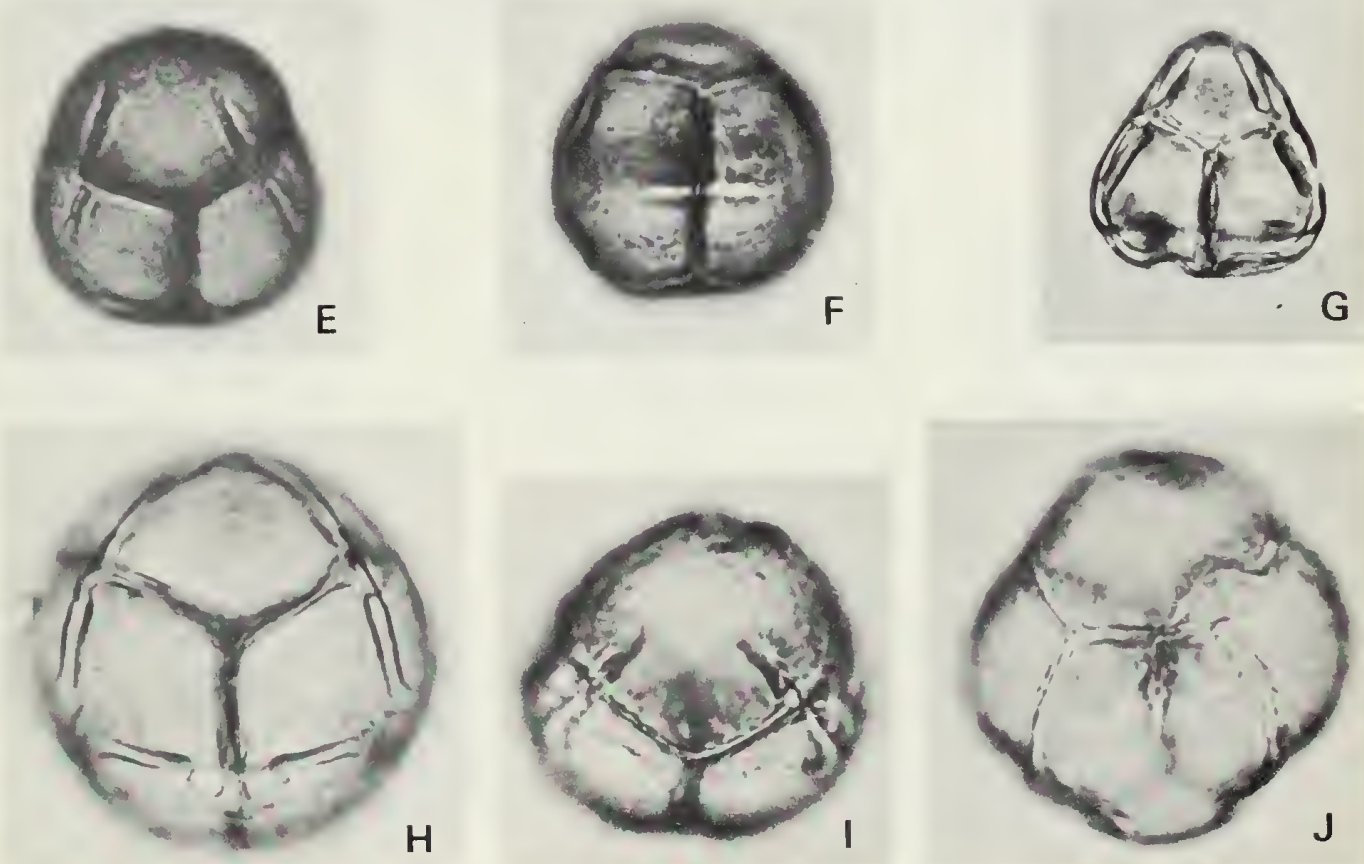
Fig. 3-Class 2, Vesiculate, X500 (p. 5)
A. Picea glauca, ev
B. P. glauca, pv, distal
C. P. mariana, ev
D. P. mariana, $\mathrm{pv}$, distal
E. Abies balsamea, ev
F. A. balsamea, pv, distal
G. Pinus banksiana, ev
H. P. banksiana, pv, distal
I. P. resinosa, ev
J. P. resinosa, ev
K. P. strobus, pv, distal 
FIGURE 3
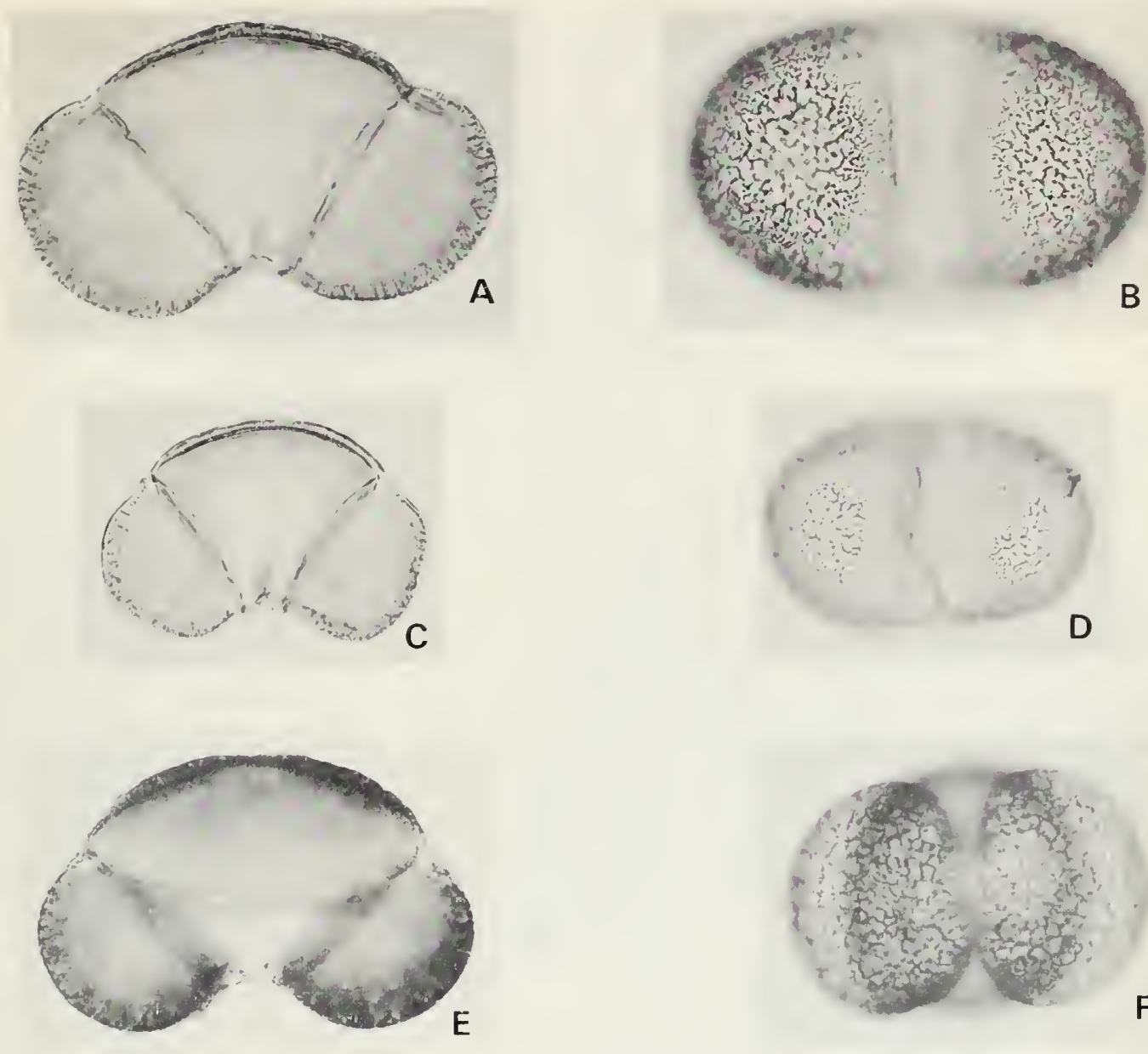

F
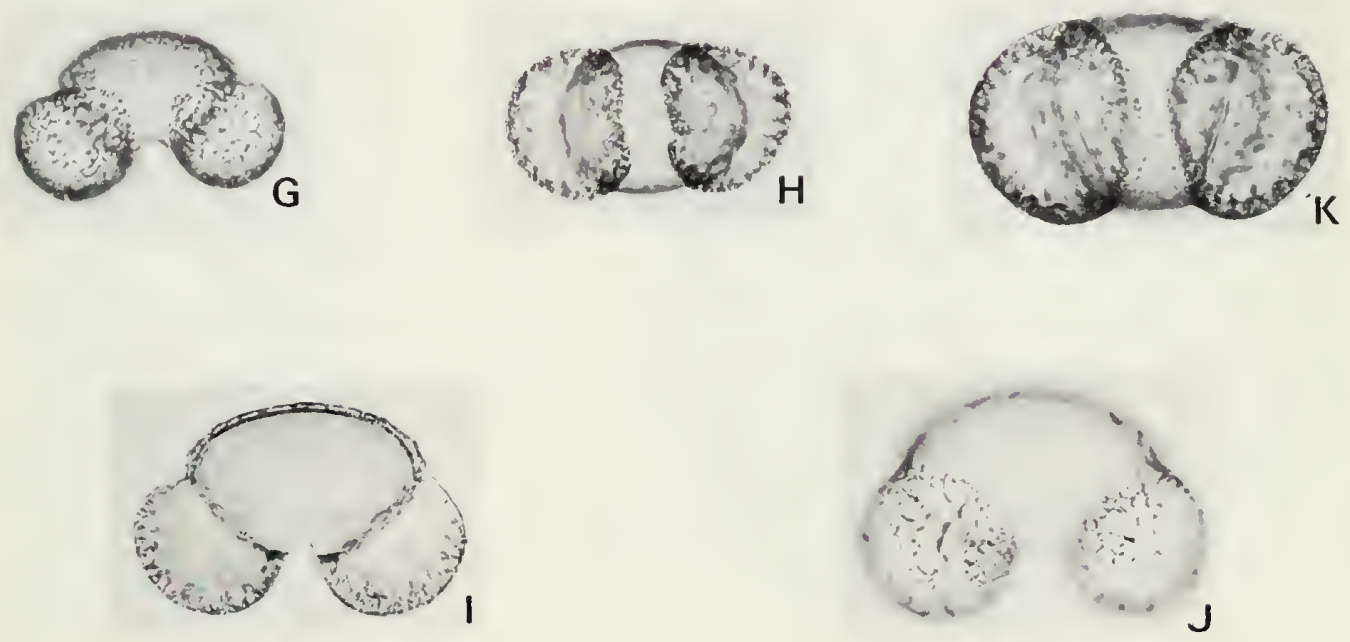
Fig. 4-Class 3, Polyplicate, X1000 (p. 5)
A. Ephedra torreyana, ev
B. E. nevadensis, ev
Class 4, Inaperturate, X1000 (p. 5)
C. Ruppia occidentalis
D. Larix laricina
E. Tsuga canadensis

$45.0(42-50) \mu \mathrm{m}$ 
FIGURE 4
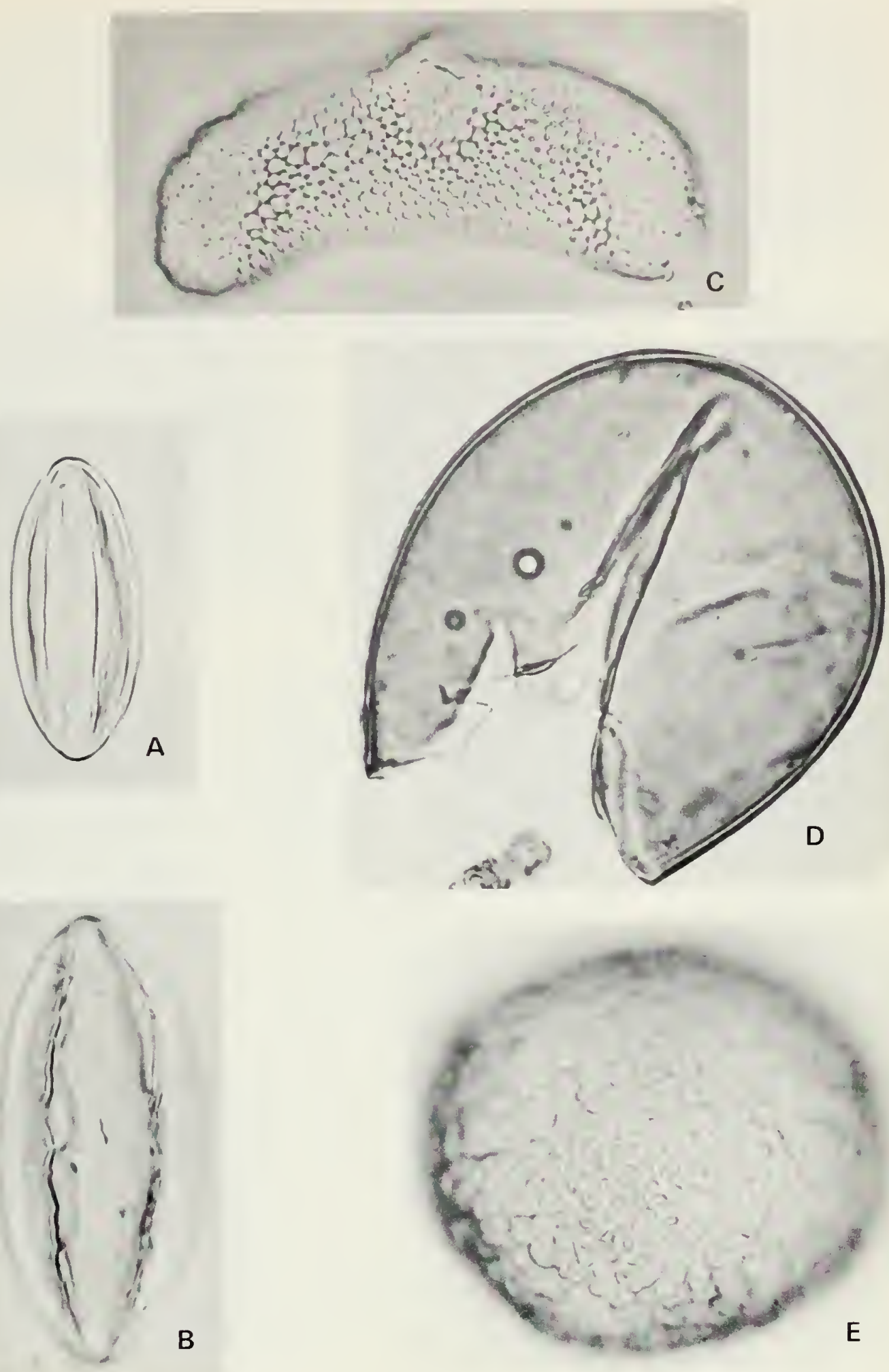
Fig. 5-Class 4, Inaperturate cont'd., X1000 (p. 5)
A. Thuja occidentalis
B. Juniperus communis
C. J. communis
D. Equisetum arvense
E. Populus tremuloides
F. Carex pensylvanica
G. Cyperus inflexus
H. Potamogeton pectinatus
I. P. natans
J. Triglochin maritima

Class 5, Monoporate, X1000 (p. 6)

K. Zizania aquatica, ev

L. Andropogon gerardi

M. Zea mays pv, distal

40.8(36-44) $\mu \mathrm{m}$

$28.8(24-32) \mu \mathrm{m}$ $42.0(40-48) \mu \mathrm{m}$ $22.0(20-24) \mu \mathrm{m}$ $36.5(32-42) \mu \mathrm{m}$ $21.0(20-22) \mu \mathrm{m}$ $22.4(20-24) \mu \mathrm{m}$

34.2(32-36) $\mu \mathrm{m}$ $39.2(34-44) \mu \mathrm{m}$ $82.6(76-88) \mu \mathrm{m}$ 
FIGURE 5

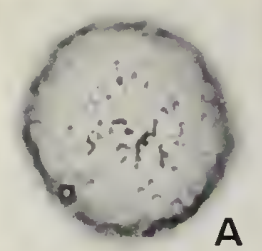

B
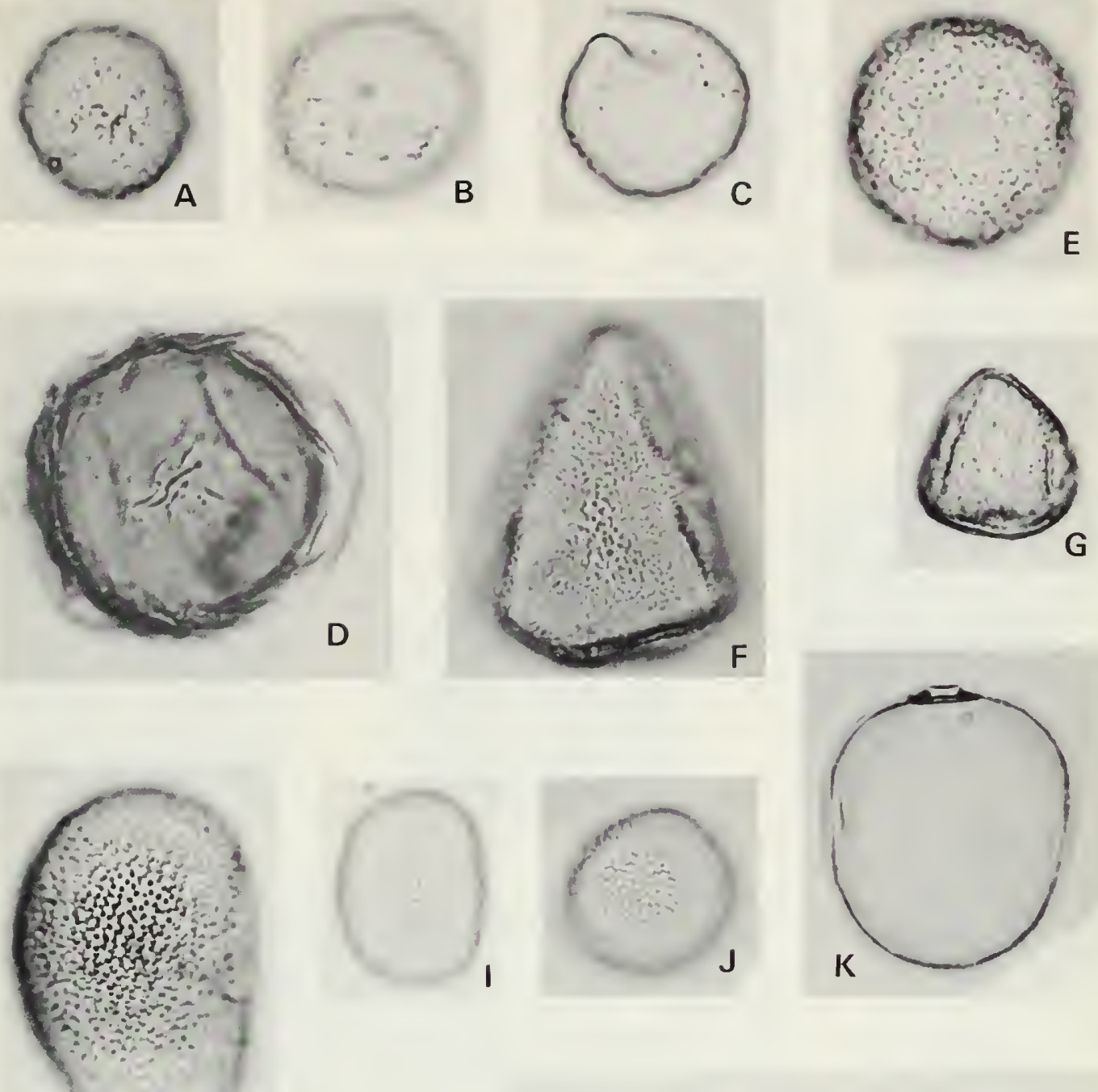

H

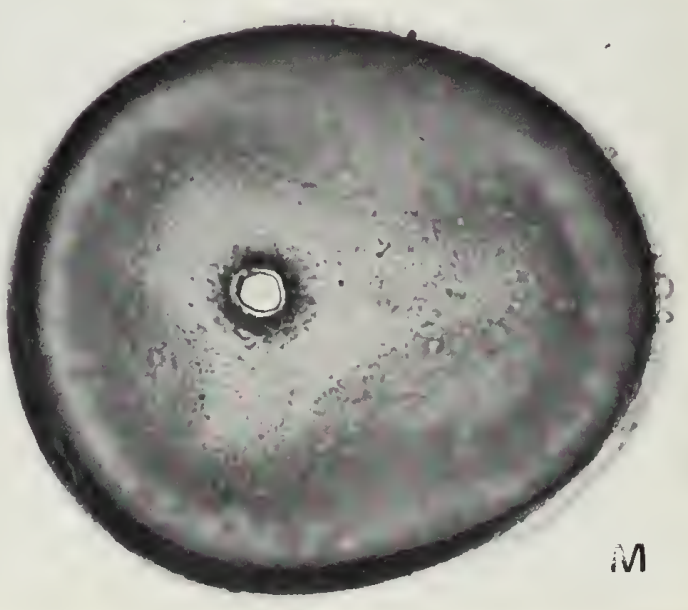


Fig. 6-Class 5, Monoporate cont'd., X1000 (p. 6)
A. Sparganium eurycarpum, pv, distal
B. Typha angustifolia, $\mathrm{pv}$, distal
C. Lemna trisulca, ev
Class 6, Diporate, X1000 (p. 6)
D. Morus rubra, ev
Class 7, Triporate, X1000 (p. 6)
E. Epilobium latifolium, pv
F. Myrica gale, pv
G. Betula papyrifera, $\mathrm{pv}$
H. B. glandulosa, pv
I. B. populifolia, pv
J. Ostrya virginiana, $\mathrm{pv}$
K. Carpinlus caroliniana, $\mathrm{pv}$
L. Tilia americana, pv
M. Huinulus lupulus, pv
N. Celtis occidentalis, $\mathrm{pv}$
o. C.occidentalis, $\mathrm{ev}$ 
FIGURE 6
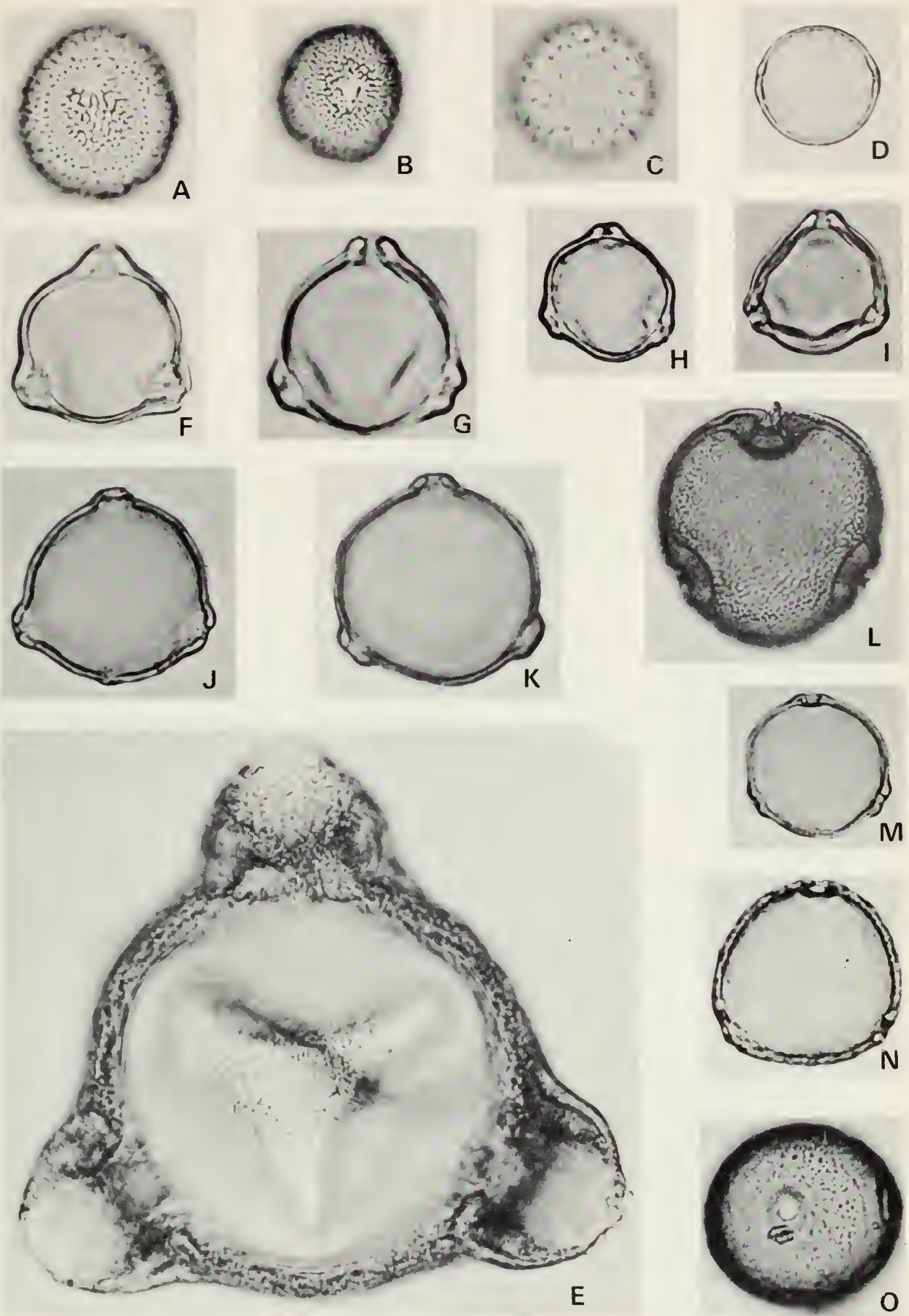
Fig. 7-Class 7, Triporate cont'd., X1000 (p. 7)
A. Carya cordiformis, pv
B. Corylus cornuta, pv
C. Urtica procera, pv
D. Morus rubra, pv

Class 8, Stephanoporate, X1000 (p. 7)

E. Alnus rugosa, pv

F. A. rugosa, pv

G. A. crispa, pv

H. Ulmus thomasii, ev

I. U. thomasii, pv

J. Myriophyllum alterniflorum, pv

K. M. exalbescens, pv Class 9, Periporate, X1000 (p. 7)

L. Eriophorum angustifolium

M. Carex lacustris

N. Juglans cinerea, pv

o. J. cinerea, $\mathrm{pv}$

P. J. nigra, pv

Q. Sagittaria latifolia

44.4(42-46) $\mu \mathrm{m}$ $22.4(22-24) \mu \mathrm{m}$ $15.6(14-18) \mu \mathrm{m}$ $19.0(18-20) \mu \mathrm{m}$

$22.0(20-24) \mu \mathrm{m}$

$18.4(18-20) \mu \mathrm{m}$ $35.0(32-38) \mu \mathrm{m}$

$19.8(18-22) \mu \mathrm{m}$ $30.2(30-32) \mu \mathrm{m}$

$38.8(36-40) \mu \mathrm{m}$ 45.8(42-50) $\mu \mathrm{m}$ $38.2(36-40) \mu \mathrm{m}$

$37.4(34-40) \mu \mathrm{m}$ $24.0(22-26) \mu \mathrm{m}$ 
FIGURE 7
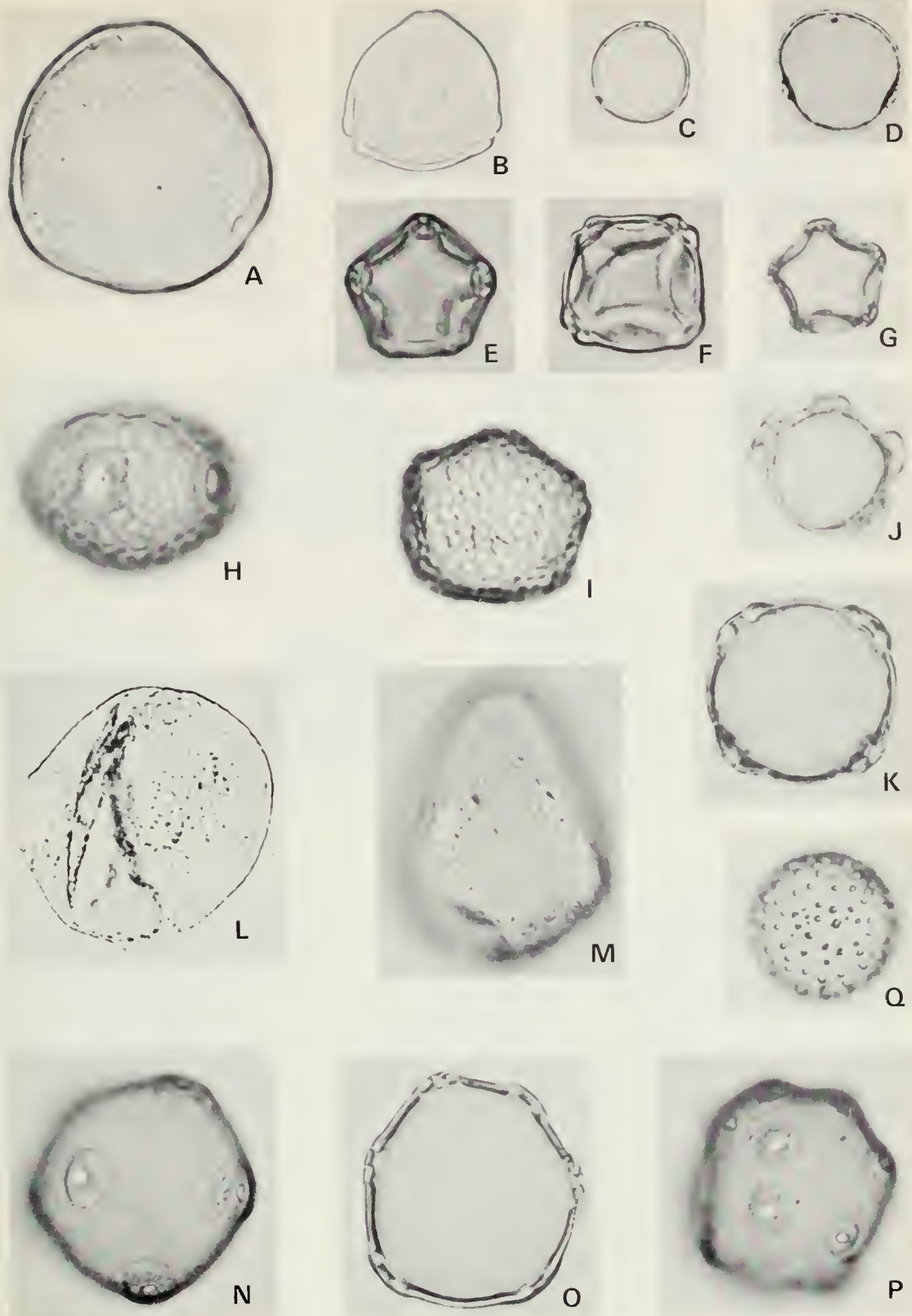

M
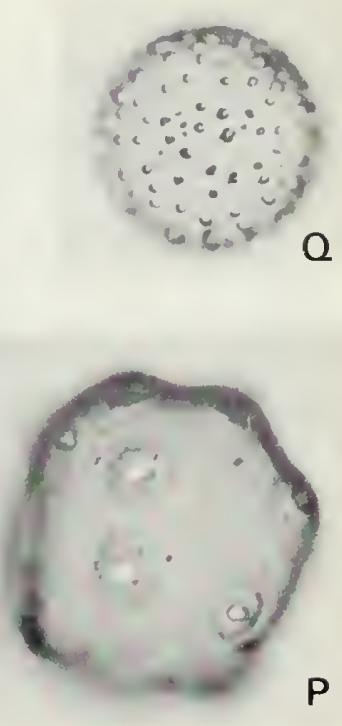
Fig. 8-Class 9, Periporate cont'd., X1000 (p. 7)
A. Polygonum lapathifolium
B. Liquidambar styraciflua
C. Stellaria crassifolia
D. Sarcobatus vermiculatus
E. Plantago lanceolata
F. Suaeda depressa
G. Salsola pestifer
H. Thalictrum dasycarpum
I. Plantago major
Class 10, Monocolpate, X1000 (p. 8)
J. Nymphaea sp., ev
K. Nymphaea sp.,pv
L. Nuphar variegatum, pv
M. Brasenia schreberi, ev
Class 11, Dicolpate, X1000 (p. 8)
N. Heteranthera dubia

$34.8(32-38) \mu \mathrm{m}$

$34.4(32-36) \mu \mathrm{m}$

$28.0(24-32) \mu \mathrm{m}$

$25.6(24-28) \mu \mathrm{m}$

$24.8(22-26) \mu \mathrm{m}$

24.6(22-28) $\mu \mathrm{m}$

$26.8(24-30) \mu \mathrm{m}$

$22.8(20-26) \mu \mathrm{m}$

$21.8(20-24) \mu \mathrm{m}$

$25.8(24-28) \mu \mathrm{m}$

48.6(46-50) $\mu \mathrm{m}$ $52.0(48-56) \mu \mathrm{m}$

$63.0(50-72) \mu \mathrm{m}$ 
FIGURE 8
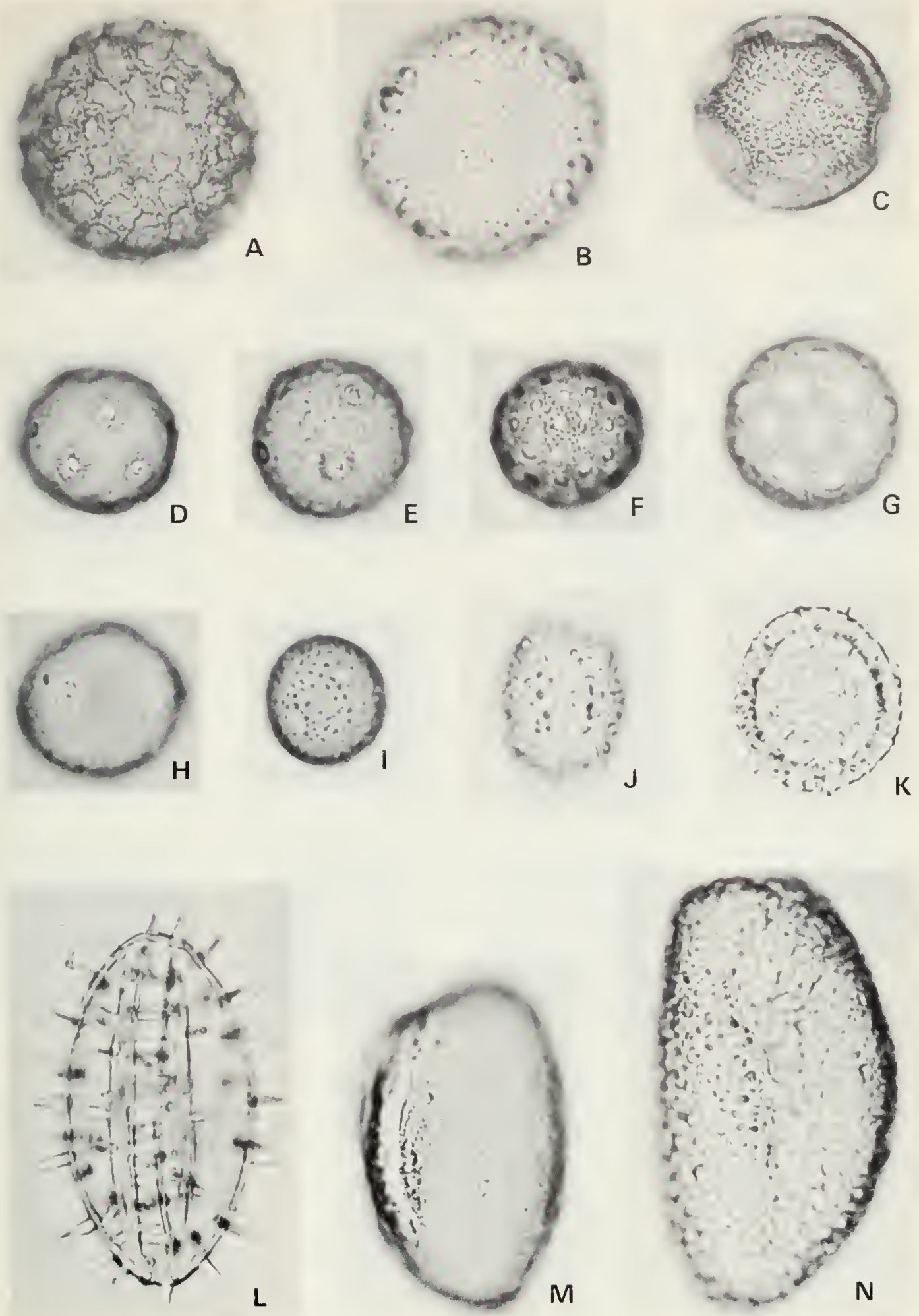
Fig. 9-Class 12, Tricolpate, X1000 (p. 8)
A. Acer rubrum, ev
B. A, rubrum, pv
C. Saxifraga oppositifolia, ev
D. S. oppositifolia, pv
E. A. saccharinum, ev
F. A. saccharinum, ev
G. Dryas integrifolia, ev
H. D. integrifolia
I. D. integrifolia, $\mathrm{pv}$
J. Acer negundo, ev
K. A. negundo, ev
L. A. negundo, $\mathrm{pv}$
M. Salix discolor, pv
N. S. discolor, ev
o. S. candida, ev
P. S. interior, ev
Q. S. herbacea, ev
R. S. herbacea, ev
S. Petalostemum purpureum, ev
T. Acer saccharum, ev
U. A. saccharum, pv 


\section{FIGURE 9}
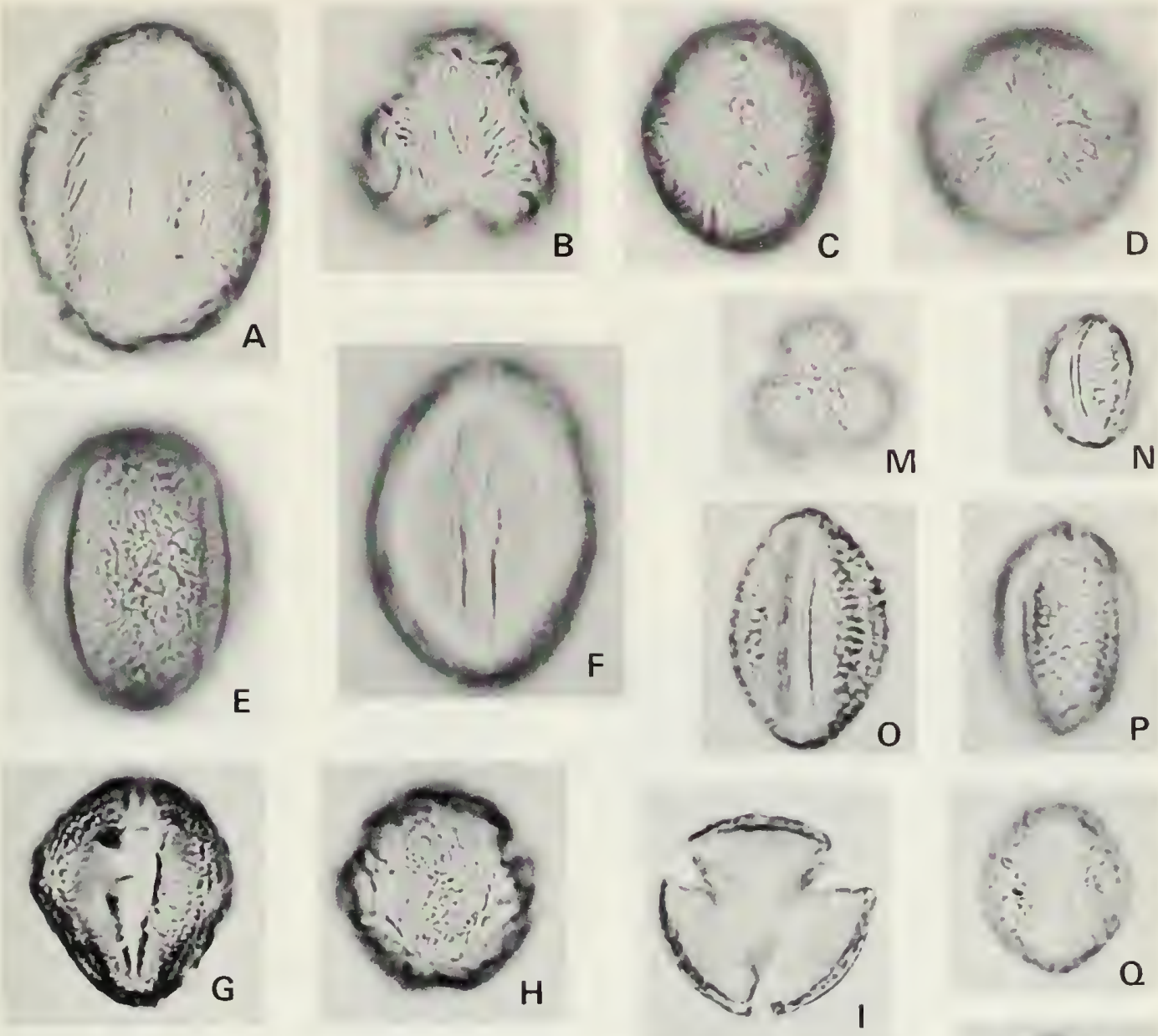

E
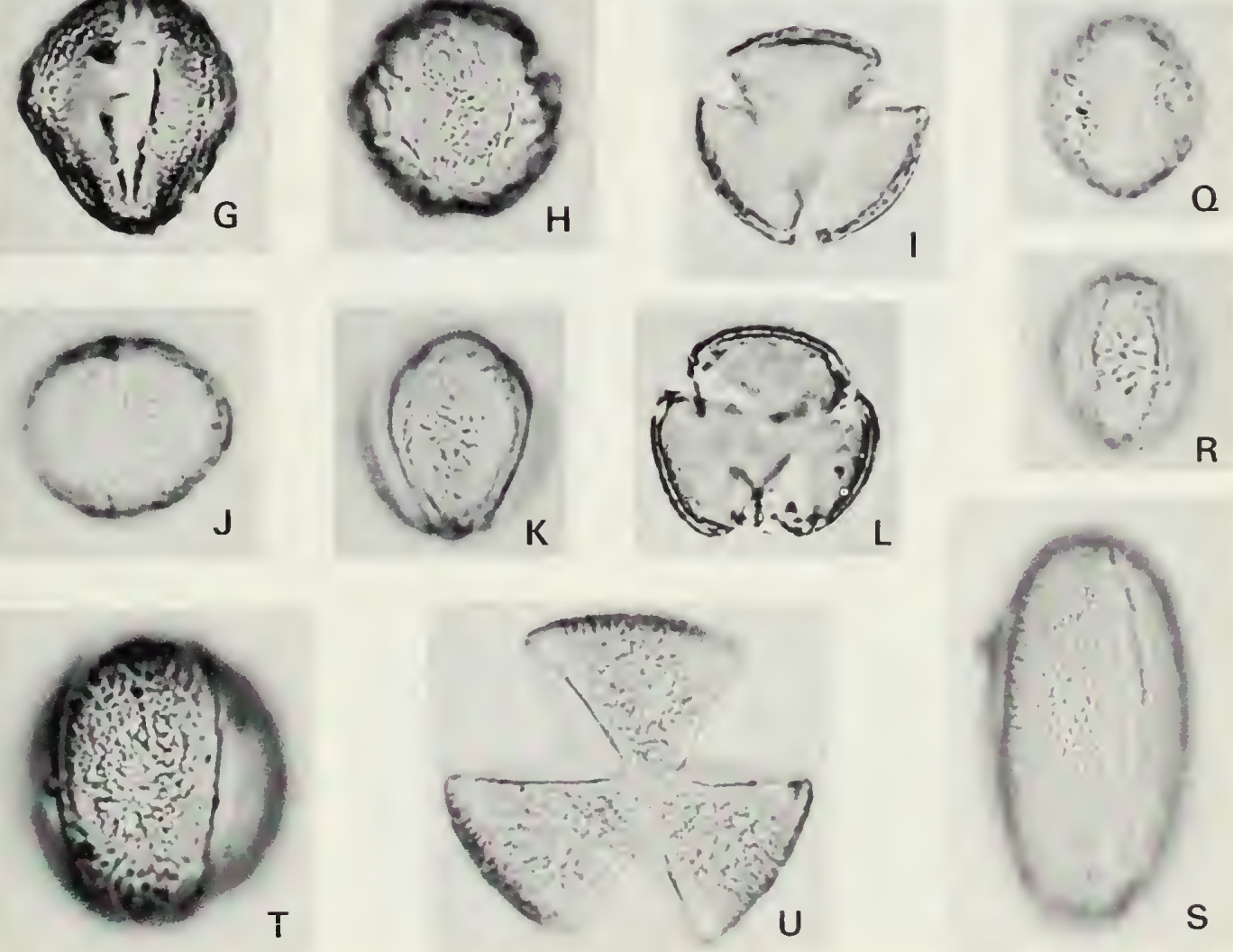
Fig. 10-Class 12, Tricolpate cont'd., X1000 (p. 9)
A. Stachys palustris, pv
B. S. palustris, pv
C. Rorippa islandica, ev
D. Brassica kaber, pv
E. Fraxinus nigra, ev
F. F. nigra, pv
G. F. quadrangulata, pv
H. Platanus occidentalis, pv
I. Quercus rubra, pv
J. Q. rubra, ev
к. Q. macrocarpa, ev
L. Caltlia palustris, ev
M. Ambrosia psilostachya, pv
N. Ranuncultus flabellaris, ev
o. Ilex verticillata, $\mathrm{pv}$
P. Nemopantluts mucronata, ev
Q. Rubus chamaemorus, pv
R. R. chamaemorus, ev

Class 13, Stephanocolpate, X1000 (p. 10)

s. Galium boreale, ev

T. G. asprellum, pv

U. G. asprellum, pv 

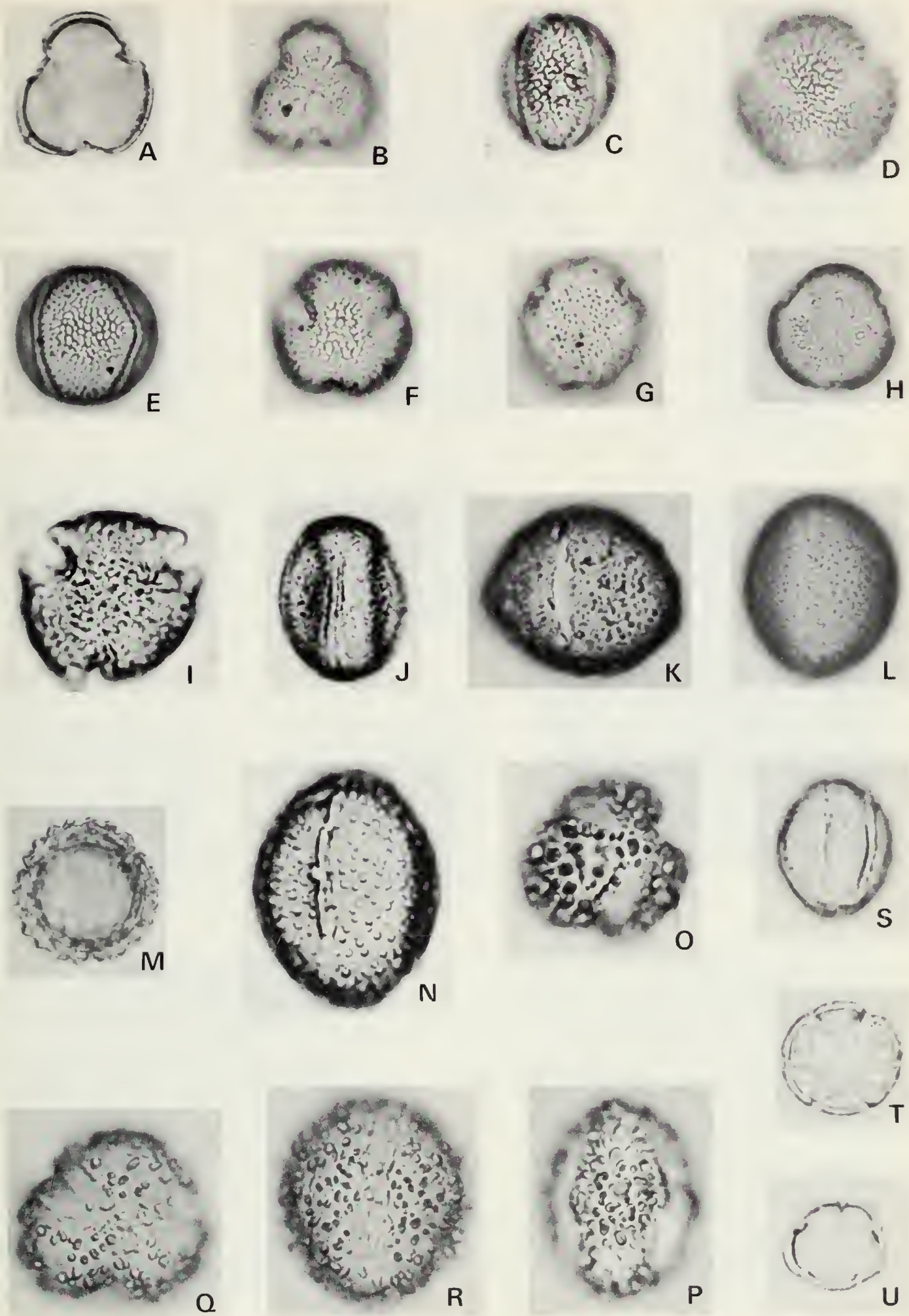
Fig. 11-Class 13, Stephanocolpate cont'd., X1000 (p. 10)
A. Hippuris vulgaris, pv
B. Lycopus virginicus, pv
C. Mentha arvensis, ev
D. Fraxinus pennsylvanica, pv
E. F. pennsylvanica, ev
F. F. americana, pv
$30.2(26-32) \mu \mathrm{m}$
$28.6(26-32) \mu \mathrm{m}$
$32.8(30-36) \mu \mathrm{m}$
$22.2(20-24) \mu \mathrm{m}$
G. Impatiens capensis, pv
26.2(24-30) $\mu \mathrm{m}$
$32.4(30-36) \mu \mathrm{m}$
H. I. capensis, pv
I. Arceuthobium pusillum, pv
$24.0(22-26) \mu \mathrm{m}$
J. A. pusillum, pv
Class 14, Pericolpate, X1000 (p. 10)
к. Polygonum amphibium
L. P. coccinium
M. Portulaca oleracea
50.2(46-54) $\mu \mathrm{m}$
$59.8(52-66) \mu \mathrm{m}$
56.8(54-64) $\mu \mathrm{m}$ 

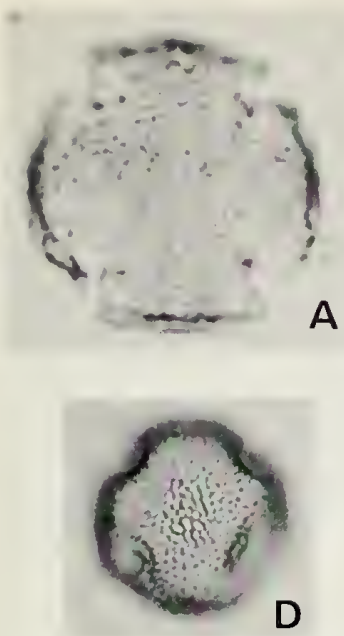
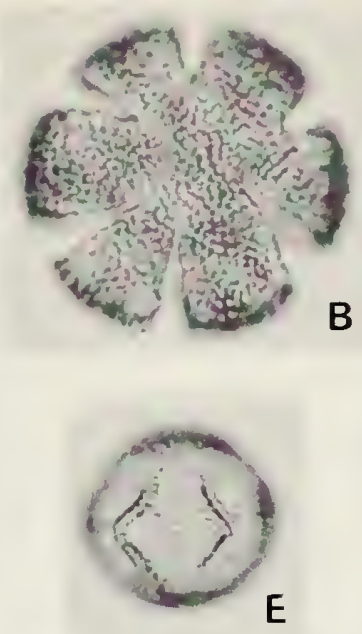
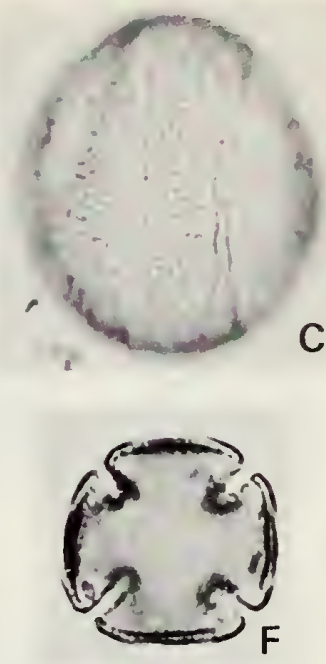
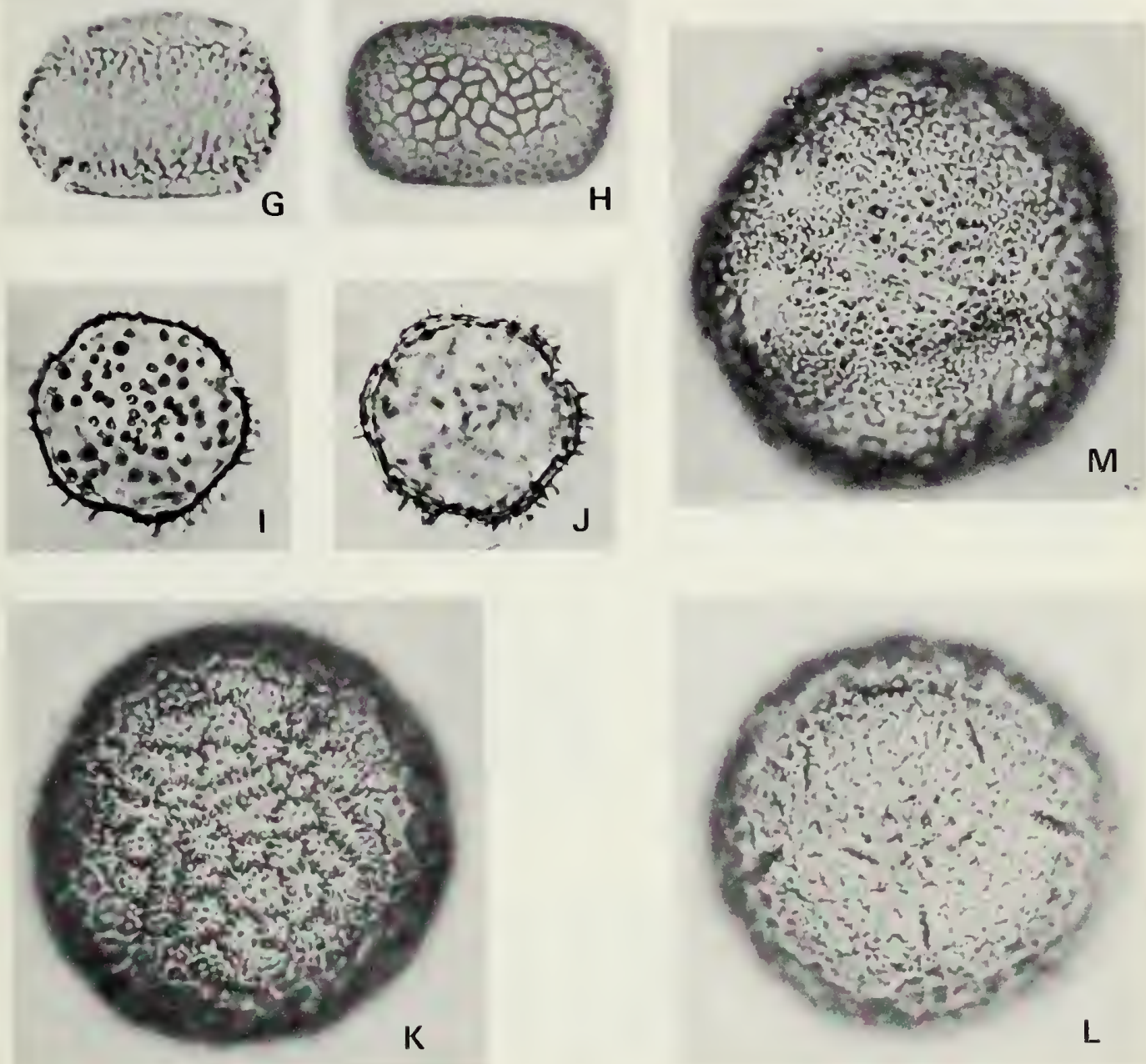
Fig. 12-Class 15, Tricolporate, X1000 (p. 10)
A. Agoseris glauca, ev
B. A. glauca, pv
C. A. glauca, pv
D. Helianthus laetiflorus, ev
E. H. laetiflorus, pv
F. Eupatorium perfoliatum, ev
G. E. perfoliatum, pv
H. Iva ciliata, $\mathrm{pv}$
I. I. ciliata, $\mathrm{pv}$
J. Xanthium pensylvanicum, pv
K. Iva xanthifolia, ev
L. I. xanthifolia, $\mathrm{pv}$
M. Ambrosia artemisiifolia, ev
N. A. artemisiifolia, pv
o. Castanea dentata, ev
P. C. dentata, $\mathrm{ev}$

$42.4(38-50) \mu \mathrm{m}$

$33.0(30-36) \mu \mathrm{m}$

$18.0(16-20) \mu \mathrm{m}$

$20.8(20-22) \mu \mathrm{m}$

$25.0(24-26) \mu \mathrm{m}$

$15.8(14-16) \mu \mathrm{m}$

$18.0(16-20) \mu \mathrm{m}$

$13.0(12-14) \mu \mathrm{m}$ 

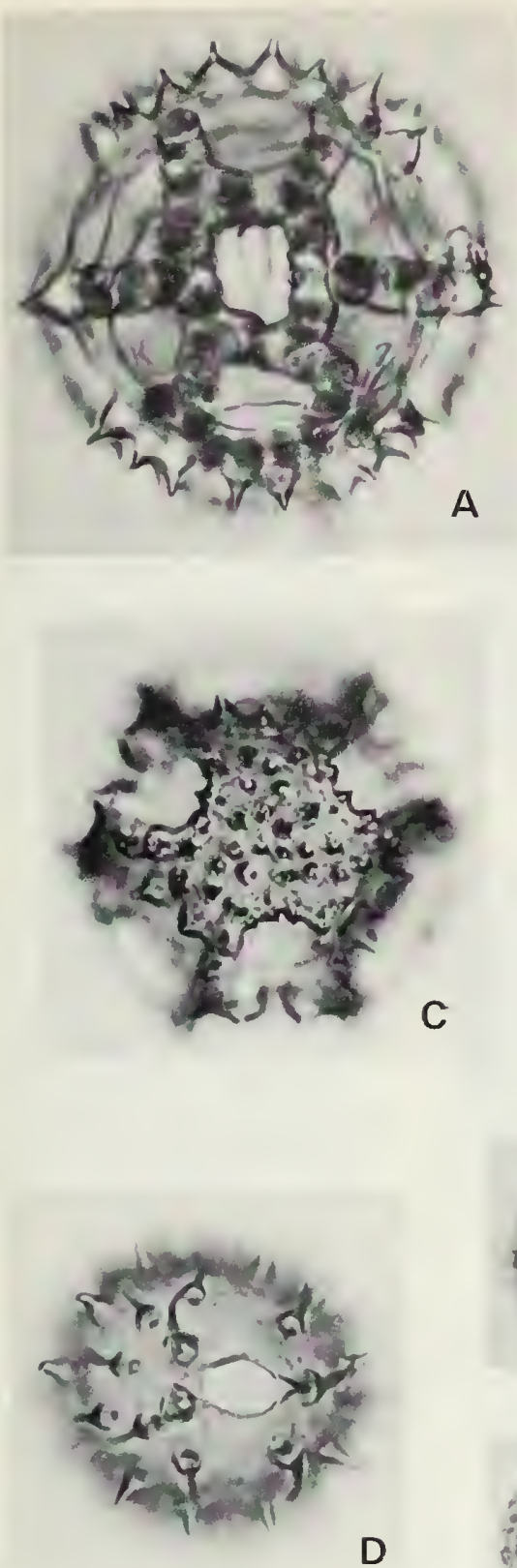
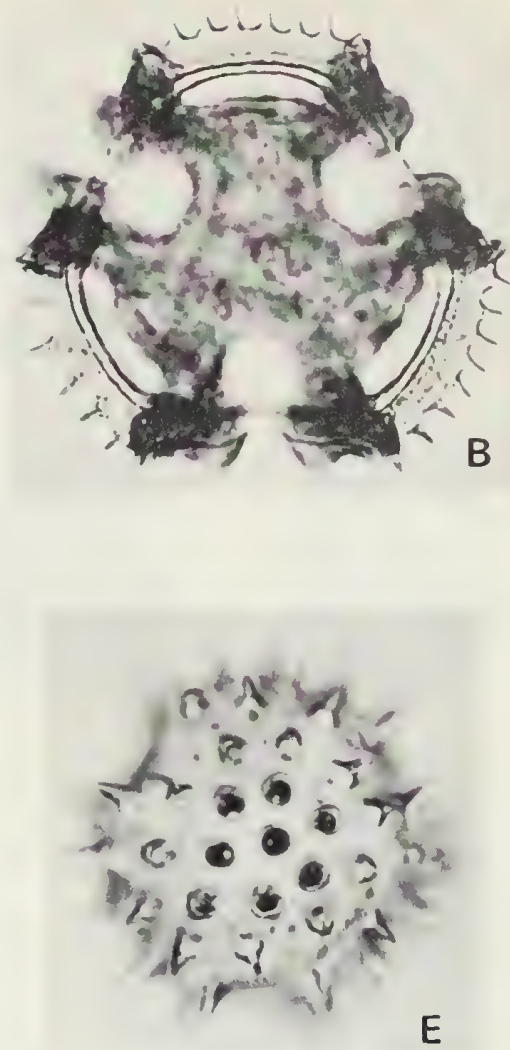

E
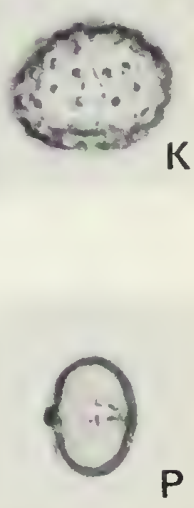
Fig. 13-Class 15, Tricolporate cont'd., X1000 (p. 11)
A. Elaeagnus commutata, pv
B. Shepherdia argentea, pv
C. Polygonum aviculare, ev
D. P. cristatum, ev
E. Artemisia ludoviciana, pv
F. A. ludoviciana, ev
G. Nyssa sylvatica, ev
H. N. sylvatica, ev
I. N. sylvatica, ev
J. Fagus grandifolia, ev
K. F. grandifolia, pv
L. Rumex mexicanus, ev
M. R. mexicanus, pv
N. Shepherdia canadensis, ev
o. S. canadensis, ev
P. Cornus canadensis, ev
Q. C. canadensis, ev

$35.8(30-40) \mu \mathrm{m}$

$34.8(30-40) \mu \mathrm{m}$

$28.8(26-32) \mu \mathrm{m}$

26.6(26-28) $\mu \mathrm{m}$

$23.0(22-26) \mu \mathrm{m}$

$31.6(30-34) \mu \mathrm{m}$

$41.2(40-44) \mu \mathrm{m}$

$25.4(24-30) \mu \mathrm{m}$

$35.8(34-38) \mu \mathrm{m}$

$23.4(22-26) \mu \mathrm{m}$ 
FIGURE 13
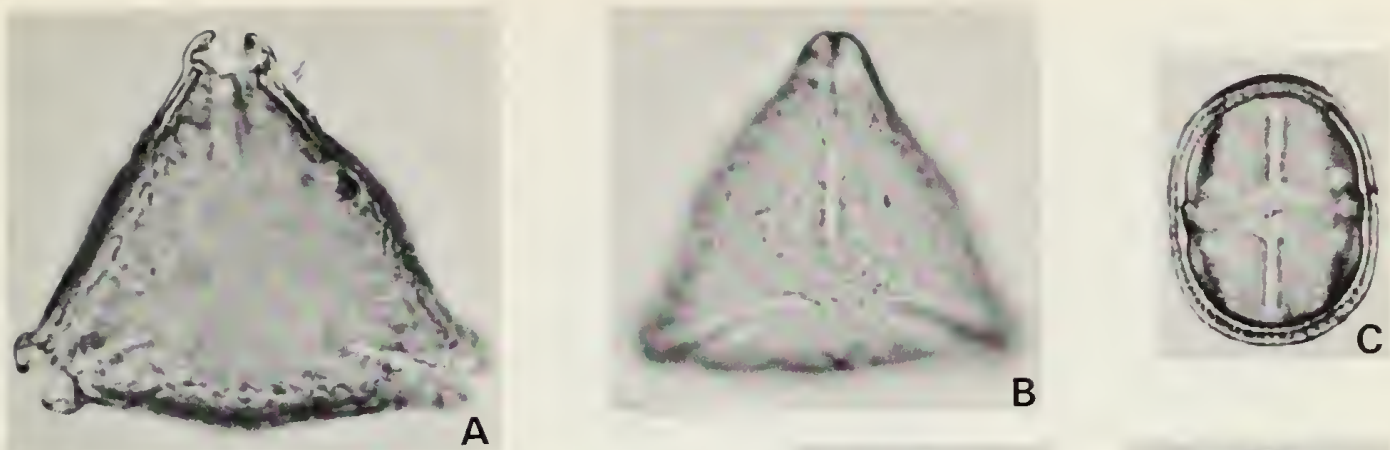

A
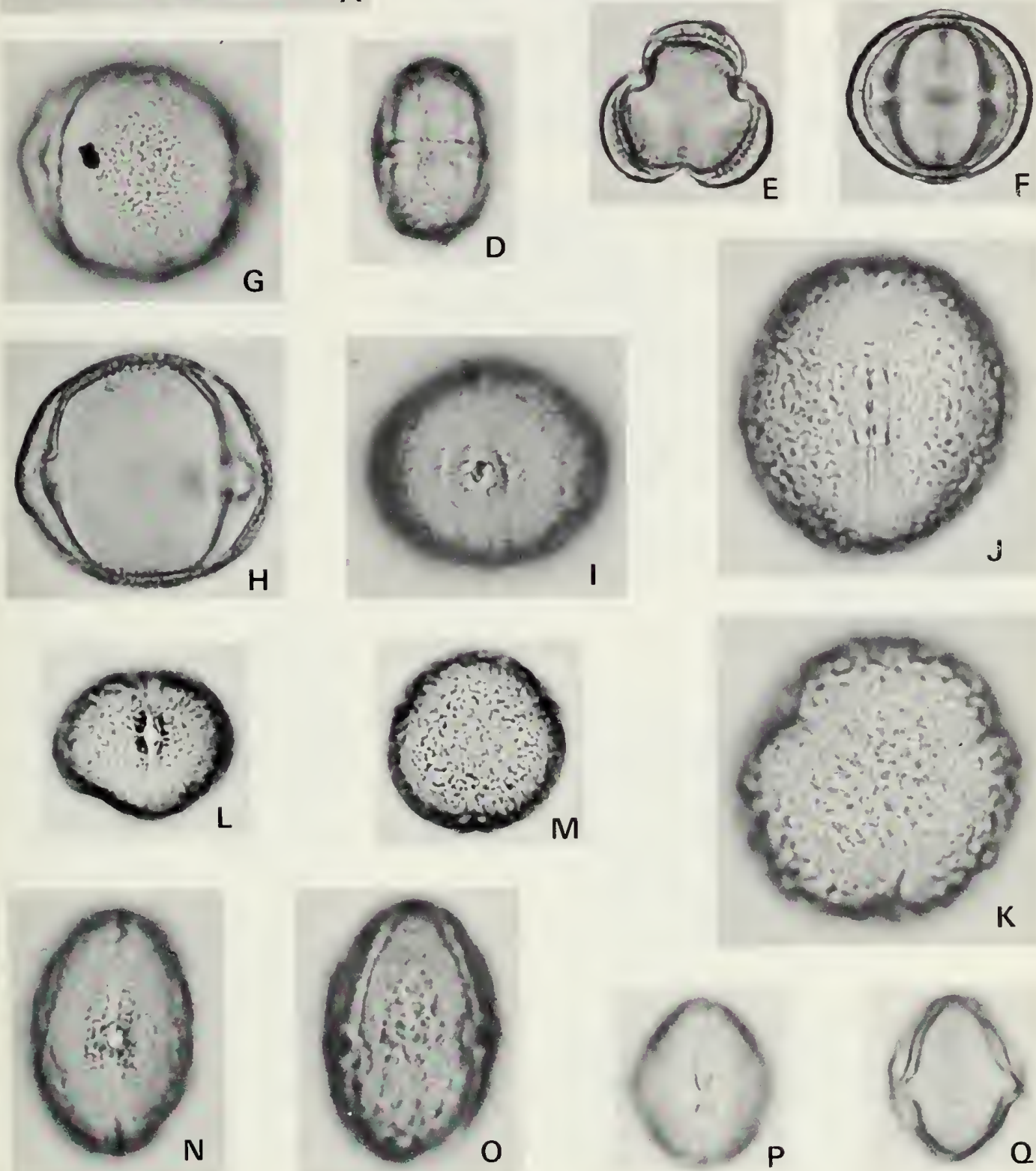

N
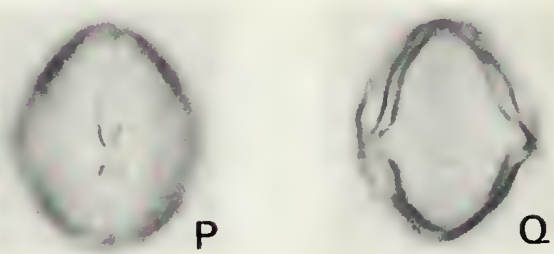
Fig. 14-Class 15, Tricolporate cont'd., X1000 (p. 11)
A. Cornus stolonifera, ev
B. C. stolonifera, ev
C. C. racemosa, ev
D. Polygonum viviparum, ev
E. P. viviparum, ev
F. Sium suave, ev
G. S. suave, ev
H. Cicuta bulbifera, ev
I. Menyanthes trifoliata, ev
J. M. trifoliata, pv
K. Prunus pensylvanica, ev
L. Potentilla palustris, ev
M. Tilia americana, ev
N. Amorpha canescens, ev
o. A. canescens, ev
P. Salix amygdaloides, ev
Q. S. amygdaloides, ev

$29.4(26-32) \mu \mathrm{m}$ $20.0(18-22) \mu \mathrm{m}$ $36.2(34-38) \mu \mathrm{m}$ $20.8(20-22) \mu \mathrm{m}$ $20.4(20-22) \mu \mathrm{m}$ 
FIGURE 14
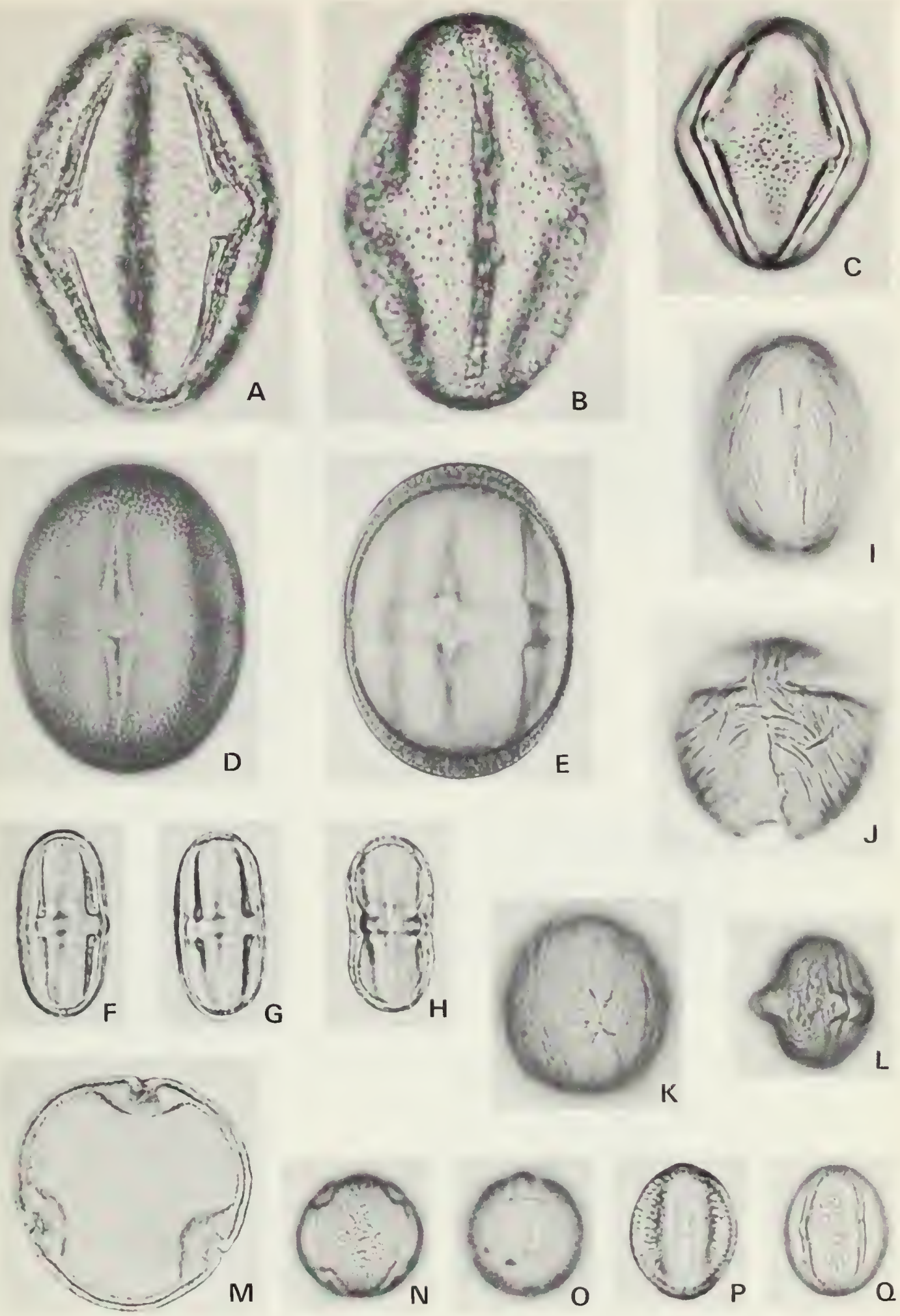
Fig. 15-Class 15, Tricolporate cont'd., X1000 (p. 12)
A. Sambucus pubens, ev
$18.6(18-20) \mu \mathrm{m}$
B. Vitis riparia, ev
20.6(20-22) $\mu \mathrm{m}$
C. V. riparia, $\mathrm{pv}$
D. Cephalanthus occidentalis, ev
$17.4(16-20) \mu \mathrm{m}$
E. C. occidentalis, ev
Class 16, Stephanocolporate, X1000 (p. 12)
F. Utricularia vulgaris, $\mathrm{pv}$
$36.4(32-40) \mu \mathrm{m}$
G. U. vulgaris, ev
H. Sanguisorba canadensis, pv
I. S. canadensis, ev 
FIGURE 15

A
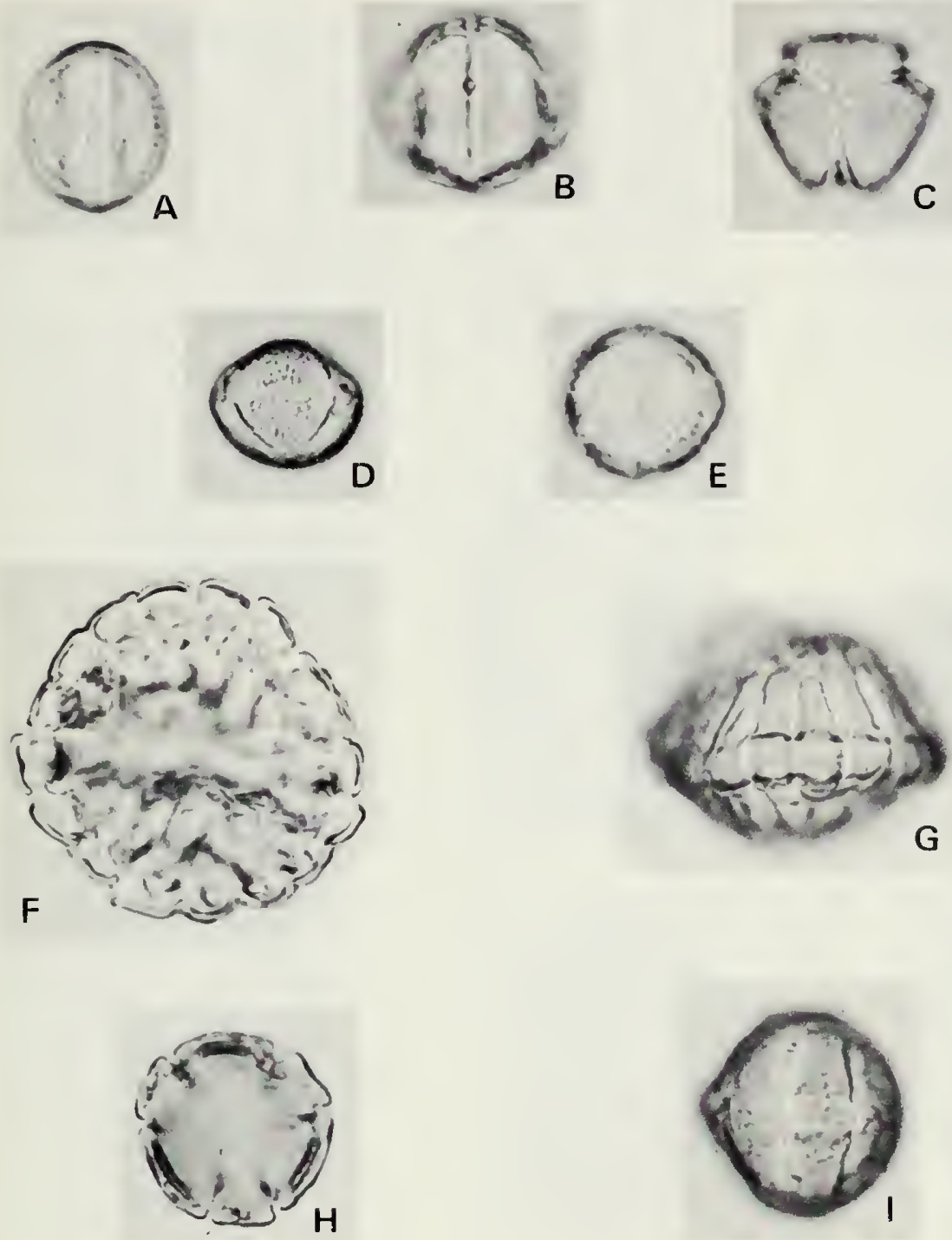
Fig. 16-Spore class 1, Alete, X1000 (p. 13)

A. Equisetum arvense

$40.8(36-44) \mu \mathrm{m}$

Spore class 2. Monolete, X1000 (p. 13)

B. Dryopteris spinulosa, ev

C. Cystopteris fragilis, pv, proximal

D. Athyrium filix-fermina, ev

E. Polypodium virginianum, ev

F. Isoetes macrospora, ev

G. Pteretis pensylvanica, ev

H. Woodsia ilvensis, ev

I. Dryopteris thelypteris, ev

J. D. goldiana, ev

K. Cystopteris bulbifera, pv, distal

L. C. fragilis, ev 
FIGURE 16
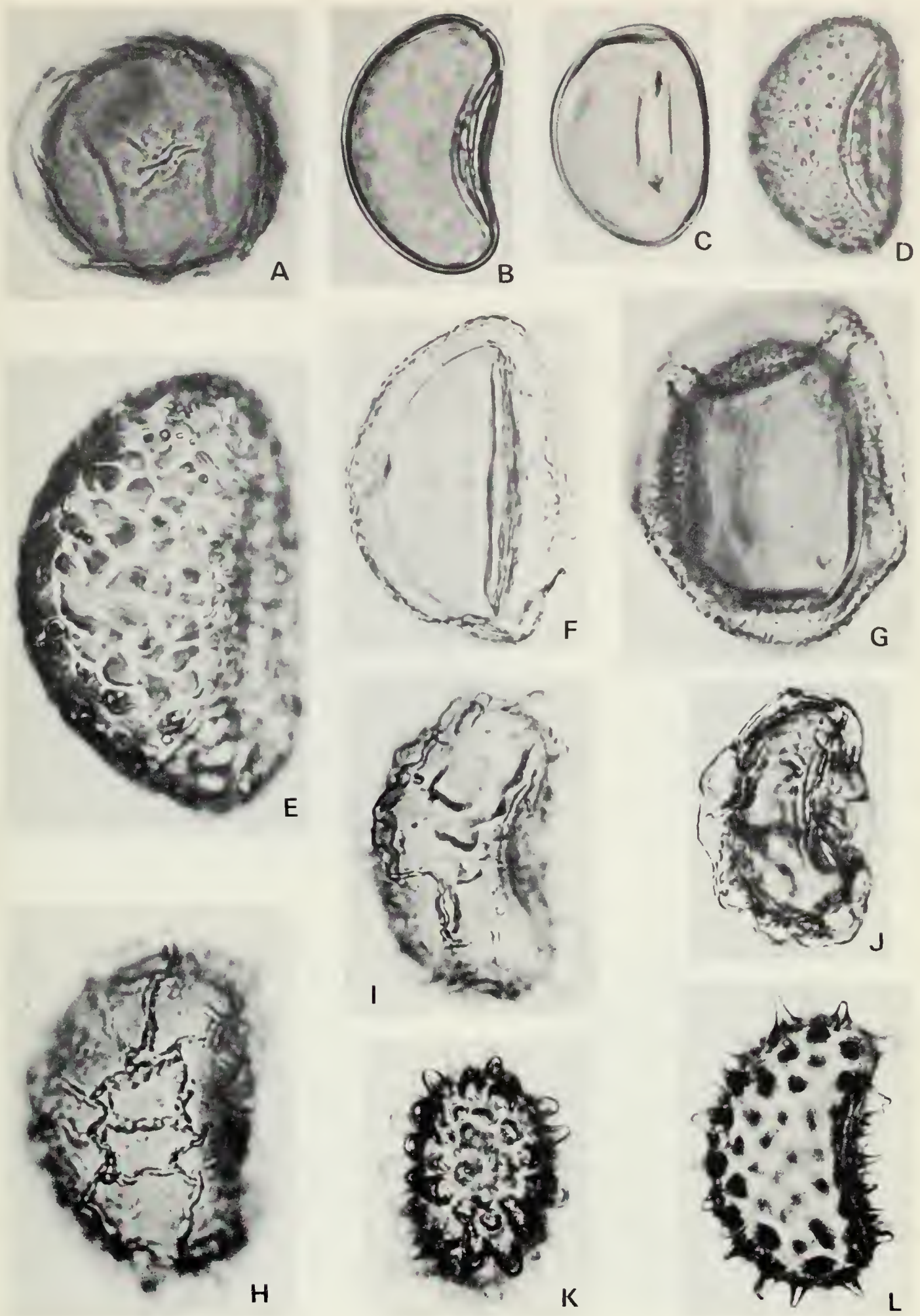
Fig. 17-Spore class 2, Monolete cont'd., X1000 (p. 13)

A. Dryopteris spinulosa, ev

$45.6(40-52) \mu \mathrm{m}$

B. Camptosaurus rhizophyllus, pv, proximal

27.0(24-32) $\mu \mathrm{m}$

C. Polystichum lonchitis, pv, distal

$35.9(32-38) \mu \mathrm{m}$

Spore class 3, Trilete, X1000 (p. 13)

D. Cheilanthes feei, pv, proximal

E. Selaginella rupestris, pv, proximal

$76.8(74-80) \mu \mathrm{m}$

$44.4(38-52) \mu \mathrm{m}$

F. Pteridium aquilinum, pv, proximal $31.2(30-36) \mu \mathrm{m}$

G. P. aquilinum, pv, distal

H. Cheilanthes feei, pv, proximal

$59.0(52-64) \mu \mathrm{m}$ 

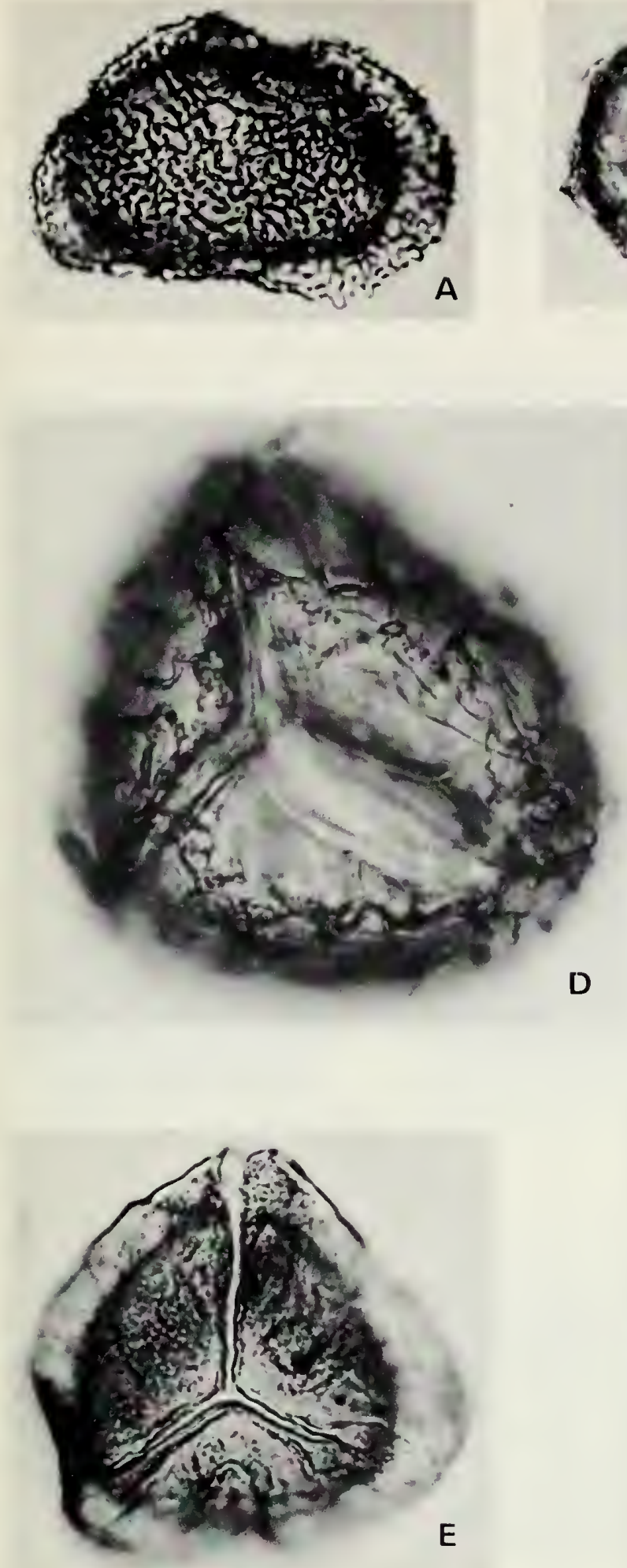
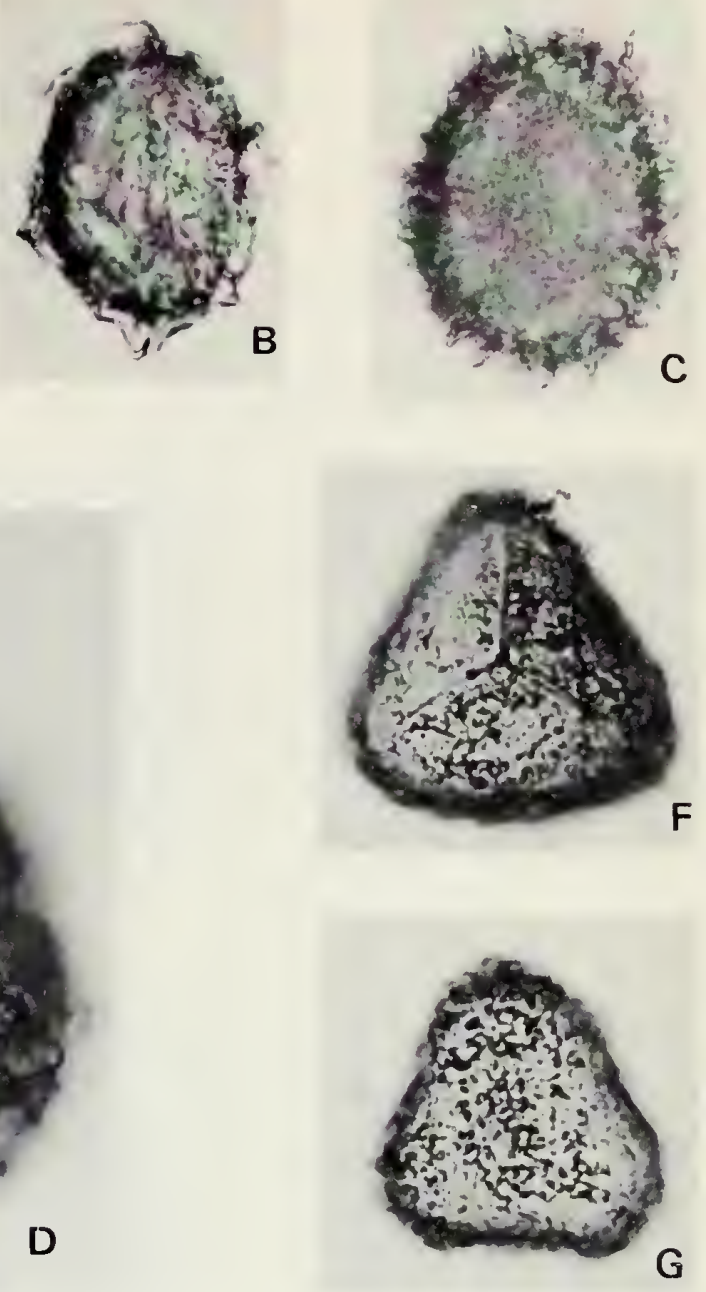

G

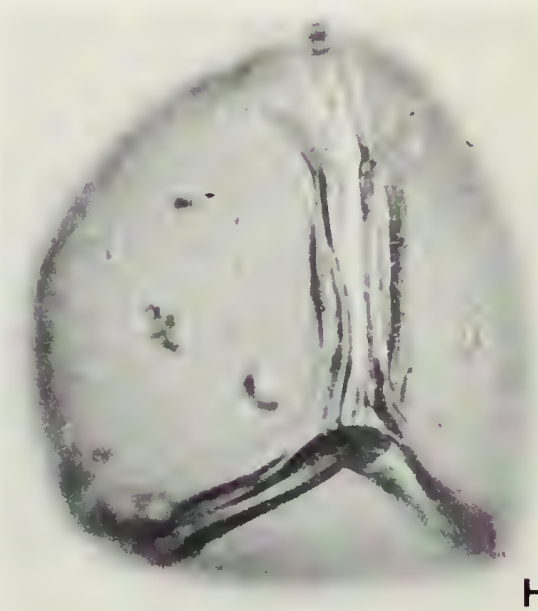


Fig. 18-Spore class 3, Trilete cont'd., X1000 (p. 14)
A. Adiantum pedatum, pv, proximal
B. Dennstaedtia punctiloba, pv, proximal
C. Pteridium aquilinum, pv, proximal
D. P. aquilinum, $\mathrm{pv}$, proximal
E. Lycopodium lucidulum, pv, distal
F. L. lucidulum, pv, proximal
G. L. selago, pv, distal
H. L. selago, $\mathrm{pv}$, proximal
I. Botrychium multifidum, pv, distal
J. B. multifidum, pv, proximal
K. B. dissectum, pv, distal
L. B. dissectum, pv, proximal 
FIGURE 18
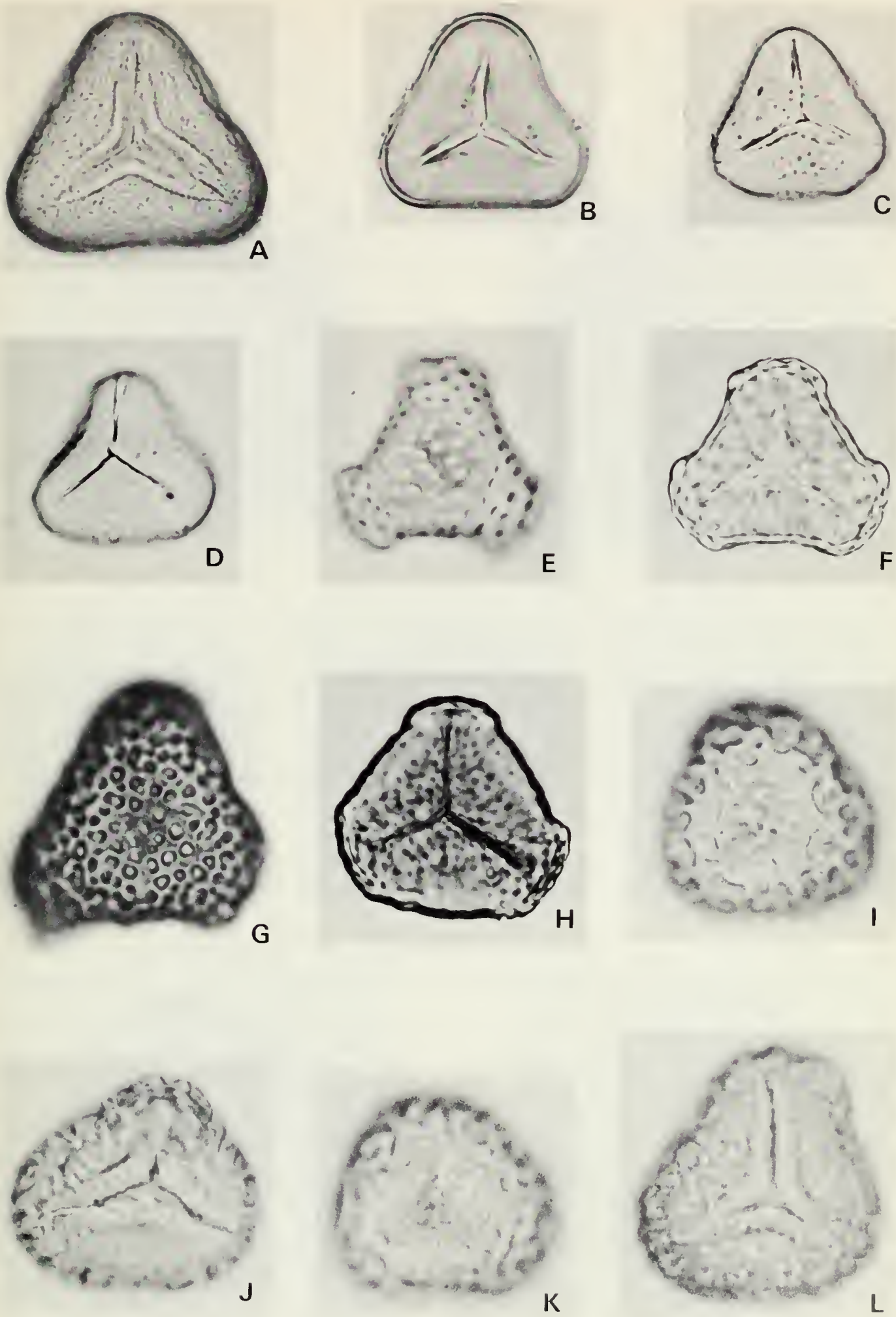
Fig. 19-Spore class 3, Trilete cont'd., X1000 (p. 14)
A. Osinunda cinnamomea, pv, distal
B. O. regalis, pv, proximal
C. O. regalis, pv, distal
D. O. claytoniana, pv, proximal
E. Selaginella selaginoides, pv, proximal
F. Lycopodilum inundatum, pv, proximal
G. L. inundatum, pv, distal

$41.2(38-44) \mu \mathrm{m}$

$51.2(48-54) \mu \mathrm{m}$

42.2(40-46) $\mu \mathrm{m}$

$32.8(30-36) \mu \mathrm{m}$ 

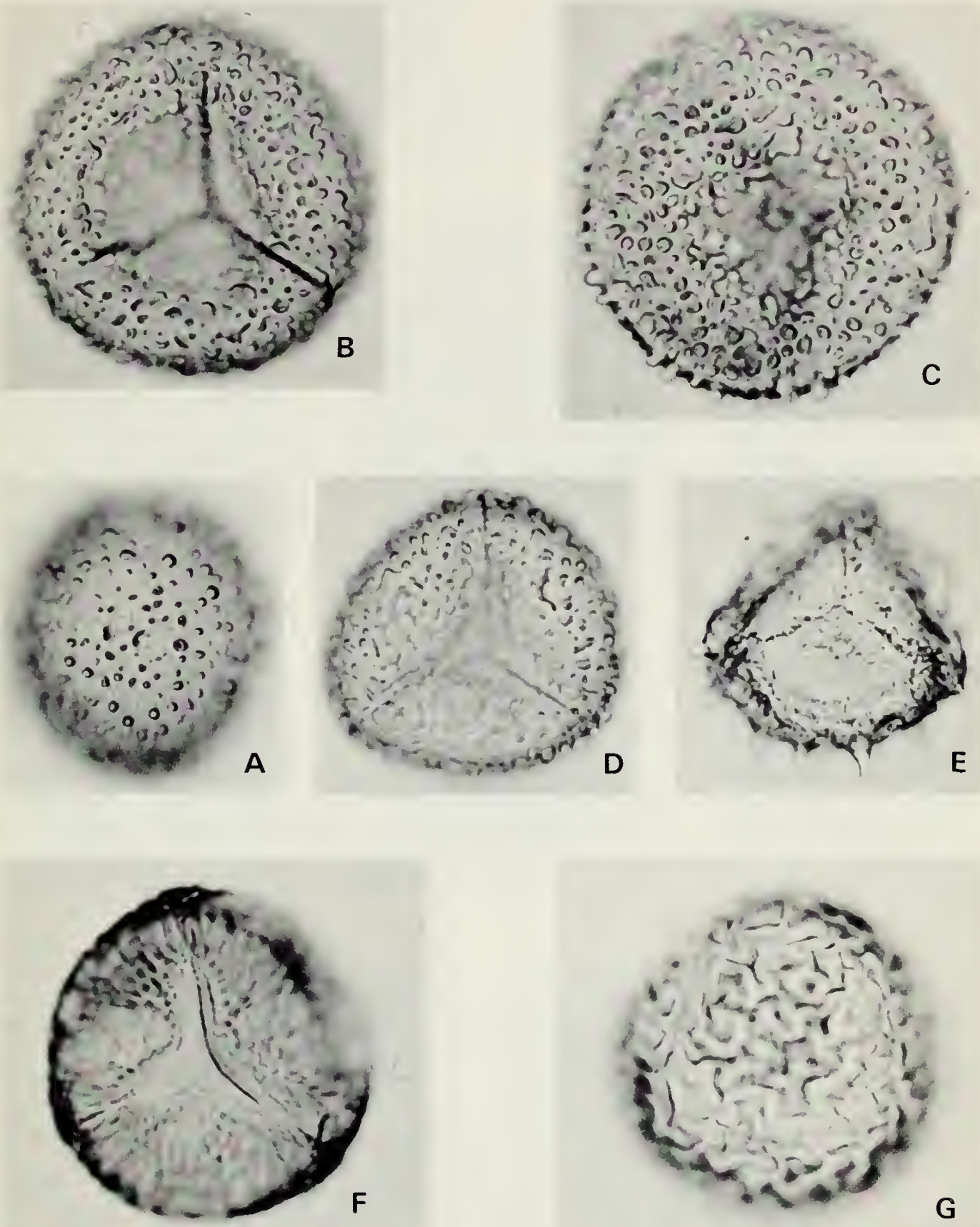
Fig. 20-Spore class 3, Trilete cont'd., X1000 (p. 14)
A. Ophioglossum vulgatum, pv, proximal
B. O. vulgatum, pv, distal
C. Lycopodium clavatum, pv, proximal
D. L. clavatum, pv, distal
E. L. complanatum, pv, proximal
F. L. complanatum, pv, distal
G. L. tristachyum, pv, proximal
H. L. tristachyum, pv, distal
I. L. obscurum, pv, proximal
J. L. obscurum, pv, distal 
FIGURE 20
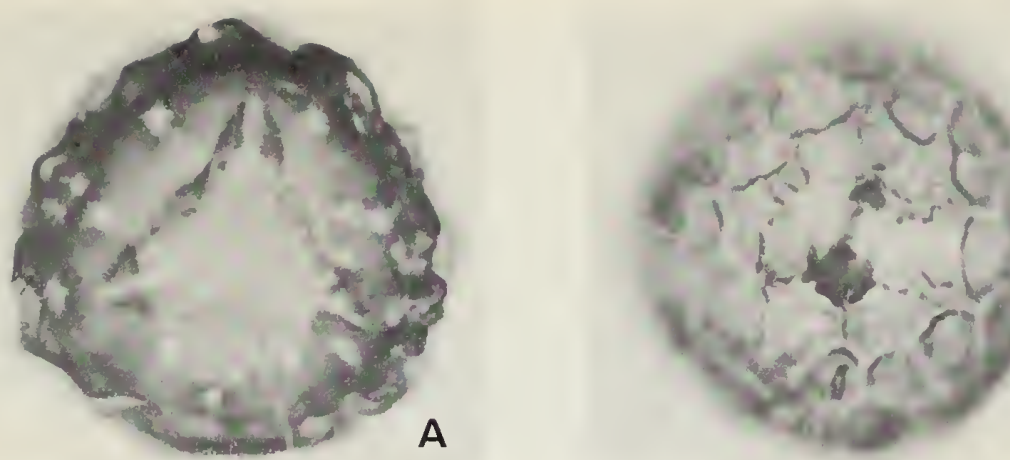

A

B
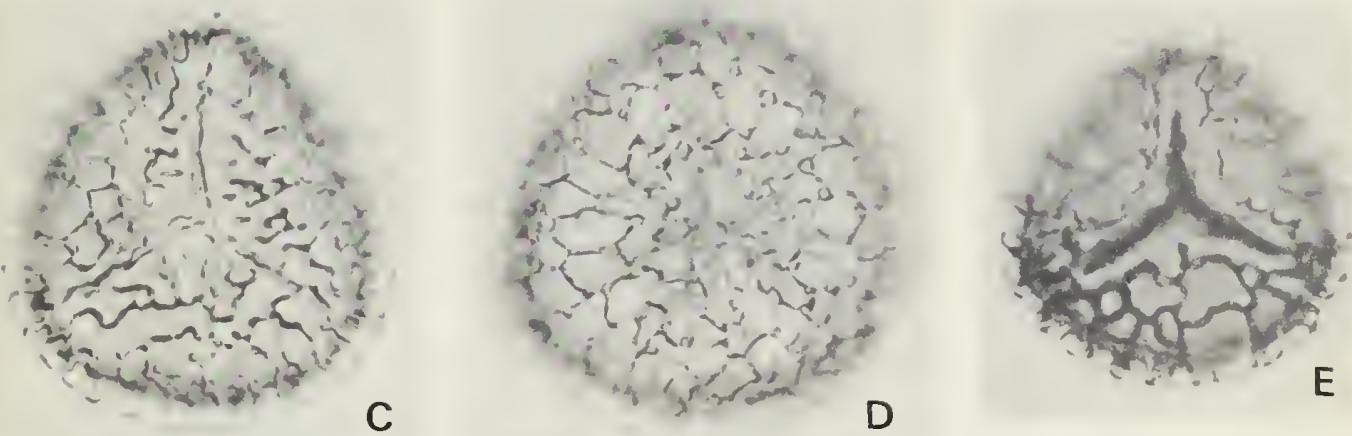

C

D

E
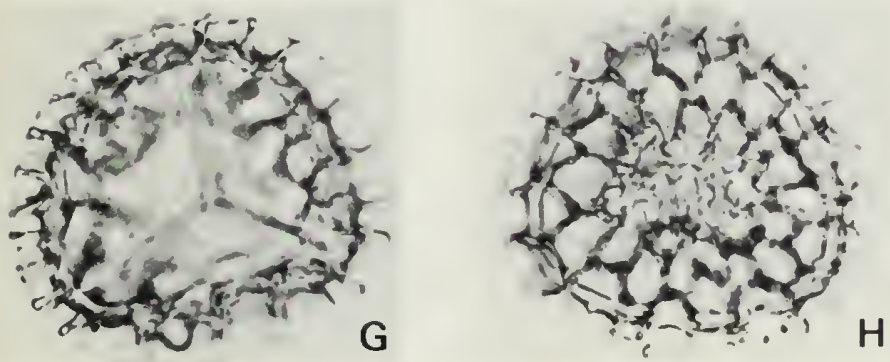

F
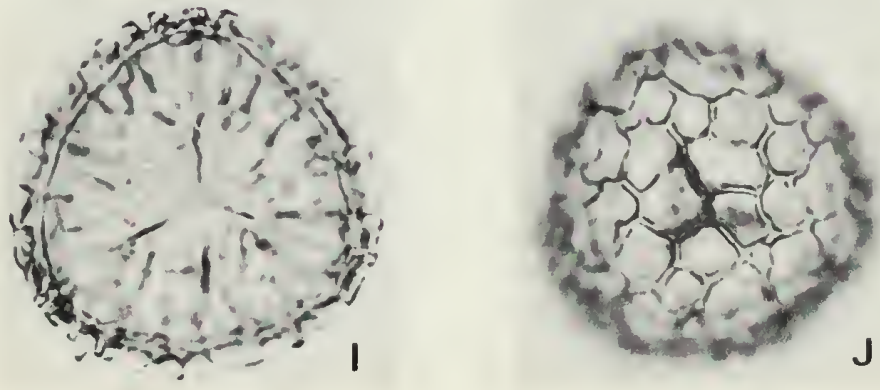
Fig. 21-Spore class 3, Trilete cont'd., X1000 (p. 15)
A. Lycopodium annotinum, pv, proximal
B. L. annotinum, pv, distal
C. Botrychium virginianum, pv, proximal
D. B. virginianum, $\mathrm{pv}$, distal
E. Sphagnum sp., pv, distal
F. Sphagnum sp., pv, proximal
G. Botrychium simplex, pv, proximal
H. B. lunaria, pv, proximal
I. B. lunaria, $\mathrm{pv}$, distal
J. B. lanceolatum, pv, proximal
K. B. lanceolatum, $\mathrm{pv}$, distal 
FIGURE 21
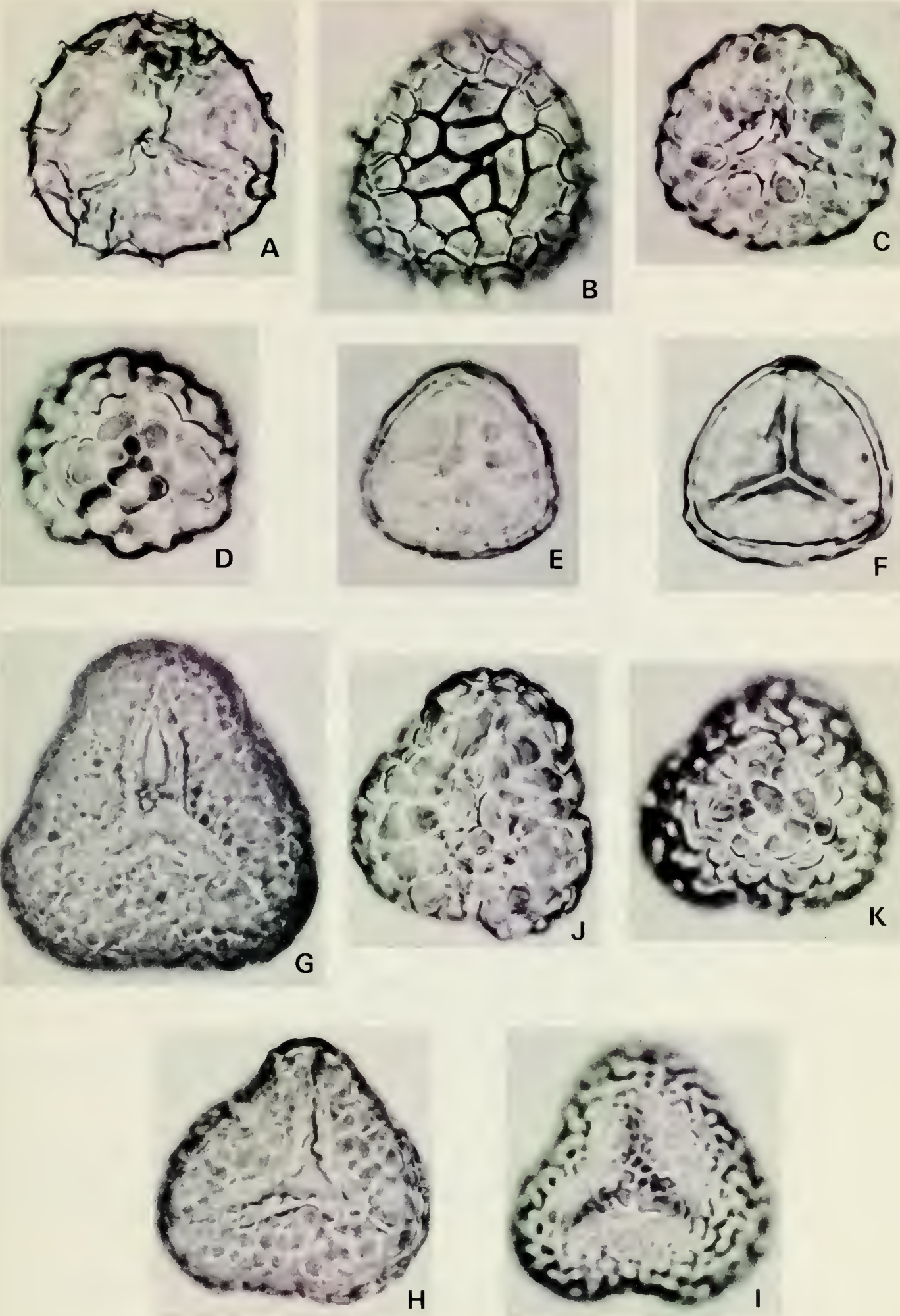


\section{Acknowledgments}

We thank Dr. J. C. Ritchie and P. Richard for critically reviewing the manuscript and supplying helpful suggestions. Miss Margaret Coutinho patiently typed the difficult key. Mr. Brian O'Donovan's skill in photographic printing was invaluable. Finally, we sincerely appreciate the comments of the several students who struggled at the miscroscope with earlier versions of the key.

Support was received through National Research Council operating grants to J. H. McAndrews (A5699) and to G. Norris (A4246).

\section{Literature Cited}

ADAMS, R. J. AND J. K. MORTON

1972 An atlas of pollen of the trees and shrubs of eastern Canada and the adjacent United States. Part 1. Gymnospermae to Fagaceae. University of Waterloo Biology Series No. 8. Waterloo, Ontario, University of Waterloo. $52 \mathrm{pp}$.

BASSETT, 1. J. AND C. W. CROMPTON

1968 Pollen morphology and chromosome numbers of the family Plantaginaceae in North America. Can. J. Bot., vol. 46, no. 4, pp. 349-361.

BRAUN, E. L.

1950 Deciduous forests of eastern North America. Philadelphia, Blakiston. $596 \mathrm{pp}$.

ERDTMAN, G.

1957 Pollen and spore morphology/plant taxonomy. Gymnospermae, Pteridophyta, Bryophyta. Stockholm, Almqvist and Wiksell. 151 pp.

1965 Pollen and spore morphology/plant taxonomy. Gymnospermae, Bryophyta. Stockholm, Almqvist and Wiksell. 191 pp.

1966 Pollen morphology and plant taxonomy. Angiosperms. Reprint ed. with addendum. New York, Hafner. (Stockholm, Almqvist and Wiksell, 1962). 553 pp.

ERDTMAN, G. AND P. SORSA

1971 Pollen and spore morphology/plant taxonomy. Pteridophyta. Stockholm, Almquist and Wiksell. $302 \mathrm{pp}$.

FAEGR1, K. AND J. IVERSON

1964 Textbook of pollen analysis. 2nd rev. ed. New York, Hafner. 237 pp.

FERNALD, M. L.

1950 Gray's manual of botany; a handbook of the flowering plants and ferns of the central and northeastern United States and adjacent Canada. New York, American Book. 1632 pp.

HARRIS, W. F.

1955 A manual of the spores of New Zealand Pteridophyta. Bull. N. Z. Dep. Scient. Ind. Res., no. 116, pp. 1-186. 
HELMICH, D. E.

1963 Pollen morphology in the maples (Acer L.). Pap. Mich. Acad. Sci., vol. 48, pt. 1, pp. 151-164.

KAPP, R. O.

1969 How to know pollen and spores. Dubuque, Iowa, W. C. Brown. $249 \mathrm{pp}$.

KREMP, G. O. W.

1965 Morphologic encyclopedia of palynology; an international, collection of definitions and illustrations of spores and pollen. Tucson, University of Arizona Press. 263 pp.

MAHER, L. J., JR.

1964 Ephedra pollen in sediments of the Great Lakes region. Ecology, vol. 45 , no. 2 , pp. 391-395.

MCANDREWS, J. H. AND A. R. SWANSON

1967 The pore number of periporate pollen with special references to Chenopodium. Rev. Palaeobotan. Palynol., vol. 3, pp. 105-117.

RICHARD, P.

1970 Atlas pollinique des arbres et de quelques arbustes indigènes du Québec. Le Naturaliste Can., vol. 97, no. 1, pp. 1-34; no. 2, pp. 97-161; no. 3, pp. 241-306.

ROWE, J. S

1959 Forest regions of Canada. Bull. For. Brch. Can., no. 123, pp. 1-71.

SEARS, P. B.

1930 Common fossil pollen of the Erie basin. Botanical Gazette, vol. 89, no. 1, pp. 95-106.

TSCHUDY, R. H. AND R. A. SCOTT (EDS.)

1969 Aspects of palynology. New York, Wiley-Interscience. 510 pp.

WHITEHEAD, D, R.

1964 Fossil pine pollen and full-glacial vegetation in southeastern North

Carolina. Ecology, vol. 45, no. 4, pp. 767-777. 





\section{LIBRARY}

ROYAL ONTARIO MUSFUM 
ISBN 0-88854-149-X 